TRANSACTIONS OF THE

AMERICAN MATHEMATICAL SOCIETY

Volume 364, Number 4, April 2012, Pages 1815-1857

S 0002-9947(2011)05379-1

Article electronically published on November 8, 2011

\title{
AN EFFECTIVE LOWER BOUND FOR GROUP COMPLEXITY OF FINITE SEMIGROUPS AND AUTOMATA
}

\author{
KARSTEN HENCKELL, JOHN RHODES, AND BENJAMIN STEINBERG
}

\begin{abstract}
The question of computing the group complexity of finite semigroups and automata was first posed in K. Krohn and J. Rhodes, Complexity of finite semigroups, Annals of Mathematics (2) 88 (1968), 128-160, motivated by the Prime Decomposition Theorem of K. Krohn and J. Rhodes, Algebraic theory of machines, I: Prime decomposition theorem for finite semigroups and machines, Transactions of the American Mathematical Society 116 (1965), 450-464. Here we provide an effective lower bound for group complexity.
\end{abstract}

\section{Contents}

1. Introduction

2. Flows and lattices

2.1. Lattices and closure operators

2.2. Abstract flows

2.3. Flow lattices

2.4. Flows on automata

3. The Presentation Lemma: Flow form 1832

3.1. Inevitable set-partitions 1837

4. The flow monoid 1838

4.1. Loopable elements 1838

4.2. Values and inevitable flows 1842

4.3. The values lemma 1844

4.4. The $n$-flow monoid 1846

4.5. The $\mathcal{F}_{n}$-operator 1847

5. The lower bound 1849

5.1. The evaluation monoid $\quad 1849$

5.2. Action on sets $\quad 1850$

5.3. The lower bound theorem 1854

6. Example: The Tall Fork 1855

References $\quad 1856$

Received by the editors December 4, 2008 and, in revised form, May 11, 2010.

2010 Mathematics Subject Classification. Primary 20M07.

Key words and phrases. Krohn-Rhodes complexity.

The second and third authors gratefully acknowledge the support of NSERC and the Committee on Research of the Academic Senate of the University of California at Berkeley for their generous support.

(C)2011 American Mathematical Society Reverts to public domain 28 years from publication 


\section{INTRODUCTION}

One of the oldest open problems in finite semigroup and automata theory is the computability of group complexity [16]. Krohn and Rhodes proved the Prime Decomposition Theorem [15] stating that every finite semigroup divides an iterated wreath product of its simple group divisors and a certain 3-element idempotent semigroup called the "flip-flop" [30, page 224]. Recall that a semigroup is called aperiodic if all its subgroups are trivial. A semigroup $S$ is said to divide a semigroup $T$, written $S \prec T$, if $S$ is a homomorphic image of a subsemigroup of $T$. It follows from the Prime Decomposition Theorem that if $S$ is a finite semigroup, then there is a division of the form

$$
S \prec A_{n} \prec G_{n} \prec A_{n-1} \cdots \curlywedge A_{1} \prec G_{1} \prec A_{0},
$$

where the $A_{i}$ are aperiodic semigroups and the $G_{i}$ are groups (where we omit the bracketing). The group complexity (or simply complexity) of $S$ is the minimum possible value of $n$ over all wreath product decompositions (1.1). A large part of finite semigroup theory has been developed around resolving this one problem of finding an algorithm to compute complexity. For instance, Tilson's influential derived category construction [42] was introduced [39, 40] exactly to provide an accessible proof to the second author's Fundamental Lemma of Complexity [21, 23, 25], stating that complexity does not drop under aperiodic surmorphisms. Ash's celebrated solution to the second author's Type II conjecture [4, 11, and its grouptheoretic reformulation [34, grew out of an attempt to compute lower bounds for complexity [32, 33].

Despite years of sustained work, there are not many classes of semigroups for which complexity is known to be decidable. In [16], Krohn and Rhodes proved that complexity is decidable for completely regular semigroups. Tilson established that complexity is decidable for semigroups with at most two non-zero $\mathscr{J}$-classes [38]. Rhodes and Tilson extended the results of [16 to semigroups in the $\mathrm{Mal}^{\prime} \mathrm{cev}$ product LG $(\mathrm{m}) \mathbf{A}$ of local groups with aperiodic semigroups [44, 22]. Computable upper and lower bounds for complexity exist 33, 32, 18, 19, 26, 2, but all existing bounds in the literature are known not to be tight. It is also known that the complexity pseudovarieties (above level 0) are not finitely based, that is, admit no finite basis of pseudoidentities [28. For a modern comprehensive survey on group complexity, consult [30, Chapter 4]; also, Tilson's chapters of Eilenberg [9, 41, 39] contain a wealth of information on complexity. Some other sources concerning complexity include [6, 37, 21, 24, 17, 31, 23, 25, 39, 14, 29, 26.

The aim of this paper is to present a new lower bound for complexity that improves on all existing bounds in the literature. The authors have some reason to believe that these bounds may be tight; only future work will tell. This research had its origins in earlier unpublished work of the second author [27.

The paper is roughly organized as follows. First we introduce the notion of flow lattices. Then we specialize to the set-partition flow lattice associated to a group mapping monoid. Afterwards, we reformulate the Presentation Lemma [6, 35, 30] in the language of flows. We then proceed to define our lower bound. Roughly speaking, the idea is that we are searching for certain sets and partitions that arise under all flows on automata of complexity $n$. We begin with elementary examples of such sets and partitions and then apply closure operators that create bigger such 
sets and partitions. Our lower bound consists of basically all the sets and partitions we can effectively construct in this way.

The reader is referred to [30] for basic notation and definitions from finite semigroup theory; see also [17, 9, 1].

\section{Flows AND LATTiCES}

The approach of Rhodes and Tilson to regular Type II elements of an arbitrary finite semigroup [33] and of Henckell [10] (see also [12, 30]) to aperiodic pointlikes shows that calculating lower bounds for such things amounts to studying closure operators on certain lattices. In the first case, one considers the partition lattice on a regular $\mathscr{R}$-class of a semigroup [43; in the latter case, one considers the power set of a semigroup. All of these lattices are examples of what we shall call flow lattices. The intuition is that one builds lower bounds up from below resulting in a closure operator (which can also be described by intersecting closed subsets from above). So, for instance, the lower bound for the Type II semigroup is the smallest subsemigroup containing the idempotents and closed under weak conjugation. The semigroup of aperiodic pointlikes of a semigroup $S$ is the smallest subsemigroup of the power semigroup $P(S)$ containing the singletons that is closed under unioning cyclic groups. We begin by setting up our abstract formalism for flows before venturing into the lattice of interest for us.

2.1. Lattices and closure operators. A lattice $L$ is a partially ordered set such that each finite subset has a meet and a join. In particular, by considering empty meets and joins, $L$ has a top $T$ and a bottom $B$. If, in addition, $L$ has arbitrary meets and joins, then it is called a complete lattice. In a complete lattice, the meet determines the join and vice versa in the usual way. See 30] for more on lattices in the context of semigroup theory. In this paper, we shall primarily be interested in finite lattices. Any finite lattice is complete and a finite partially ordered set is a lattice if and only if it has a top and admits pairwise meets.

Definition 2.1 (Closure operator). A closure operator on a complete lattice $L$ is a function $c: L \rightarrow L$ that is order-preserving, idempotent and increasing. That is, for all $\ell_{1}, \ell_{2} \in L$ :

(1) (order-preserving) $\ell_{1} \leq \ell_{2} \Longrightarrow \ell_{1} c \leq \ell_{2} c$;

(2) (idempotent) $\ell_{1} c^{2}=\ell_{1} c$;

(3) (increasing) $\ell_{1} \leq \ell_{1} c$.

We use $\mathscr{C}(L)$ to denote the set of closure operators on $L$.

Proposition 2.2. Let $L$ be a complete lattice. Then the set $\mathscr{C}(L)$ is a complete lattice with the pointwise ordering $c_{1} \leq c_{2}$ if and only if $\ell c_{1} \leq \ell c_{2}$ for all $\ell \in L$. The meet is pointwise, i.e., is given by

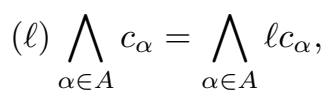

for $\ell \in L,\left\{c_{\alpha} \mid \alpha \in A\right\} \subseteq \mathscr{C}(L)$. The top of $\mathscr{C}(L)$ is the constant function taking the value $T$ (the top of $L$ ); the bottom is the identity map $1_{L}$. 
Proof. Let $\left\{c_{\alpha} \mid \alpha \in A\right\} \subseteq \mathscr{C}(L)$ and denote by $c$ the pointwise meet of this set. First of all, observe that if $\ell \leq \ell^{\prime}$ and $\beta \in A$, then

$$
\bigwedge_{\alpha \in A} \ell c_{\alpha} \leq \ell c_{\beta} \leq \ell^{\prime} c_{\beta}
$$

Thus $\bigwedge_{\alpha \in A} \ell c_{\alpha} \leq \bigwedge_{\alpha} \ell^{\prime} c_{\alpha}$ and hence $c$ is order-preserving. Next we show that $c$ is increasing. Indeed, if $\ell \in L$, then $\ell \leq \ell c_{\alpha}$, for all $\alpha \in A$ and hence $\ell \leq$ ( $\ell) \bigwedge_{\alpha \in A} c_{\alpha}=\ell c$.

Since $c$ is increasing, $\ell c \leq \ell c^{2}$. Thus we need only establish the reverse inequality. Now if $\alpha \in A$, then since $c$ is pointwise below $c_{\alpha}$ we have $\ell c^{2} \leq \ell c_{\alpha}^{2}=\ell c_{\alpha}$ and hence $\ell c^{2} \leq \bigwedge_{\alpha \in A} \ell c_{\alpha}=\ell c$. This concludes the proof that $\mathscr{C}(L)$ is closed under pointwise meets. Hence $\mathscr{C}(L)$ is a lattice and the constant map to $T$ is the top of $\mathscr{C}(L)$. Since closure operators are increasing, plainly $1_{L}$ is the bottom.

We remark that the join in $\mathscr{C}(L)$ is the determined join [30, Chapter 6] and is not in general the pointwise join. (The determined join of a subset is the meet of all its upper bounds.)

The easiest way to understand these notions is via the following alternative characterization of a closure operator. If $c$ is a closure operator on $L$, an element $\ell \in L$ is called stable or closed if $\ell c=\ell$. It is well known that the set of stable elements $L c$ is a meet-closed subset of $L$ [30, Proposition 6.3.6]. Conversely, if $K \subseteq L$ is a meet-closed subset (and so $T \in K$ ), then the function $c_{K}: L \rightarrow L$ given by

$$
\ell c_{K}=\bigwedge\{k \in K \mid \ell \leq k\}
$$

is a closure operator with $K=L c_{K}$. Moreover, if $c \in \mathscr{C}(L)$, then $c_{L c}=c$ [30, Proposition 6.3.6]. Hence from (2.1) it is immediate that

$$
c_{1} \leq c_{2} \text { in } \mathscr{C}(L) \Longleftrightarrow \operatorname{Im} c_{1} \supseteq \operatorname{Im} c_{2} .
$$

We remark that the reversal in (2.2) is crucial. The above discussion shows that if $\mathrm{C}(L)$ denotes the collection of meet-closed subsets of $L$ ordered by reverse inclusion, then $\mathrm{C}(L)$ is a complete lattice with join given by intersection. The bottom is the set $L$, the top is the set $\{T\}$. The meet is determined; namely, the meet of a subset $W$ is the intersection of all meet-closed subsets containing $W$. Equivalently, one takes the union of $W$ and then closes it under meets. Our discussion establishes the following well-known proposition.

Proposition 2.3. Let $L$ be a complete lattice. Then the complete lattices $(\mathscr{C}(L), \leq)$ and $(\mathrm{C}(L), \supseteq)$ are isomorphic.

Henceforth, we identify $\mathscr{C}(L)$ and $\mathrm{C}(L)$ and so we drop the notation $\mathrm{C}(L)$.

2.2. Abstract flows. Let $L$ be a complete lattice. Then $L^{2}=L \times L$ is a complete lattice under coordinate-wise ordering. The meet and join are coordinate-wise.

A binary relation on a set $A$ is a subset of $A \times A$. If $f$ is a binary relation, we write $a f b$ to indicate $(a, b) \in f$. Binary relations form a monoid $B(A)$ where composition is given by $x f g y$ if and only if there exists $z \in A$ so that $x f z g y$ for $x, y \in A$ and $f, g \in B(A)$. The identity $I_{A}$ is just the diagonal $\{(a, a) \mid a \in A\}$. Sometimes, it is convenient to identify $f \in B(A)$ with the map $f^{\prime}: A \rightarrow P(A)$ 
(the power set of $A$ ) given by $a f^{\prime}=\{b \in A \mid a f b\}$. In particular, we will abuse notation and denote the function and the relation by the same letter. Consequently, any partial function $f: A \rightarrow A$ can be viewed as a binary relation. The associated subset of $A \times A$ is the graph of $f$, i.e., the set $\{(a, f(a)) \mid a \in \operatorname{dom} f\}$. For instance, the identity of $B(A)$ is the binary relation corresponding to the identity function on $A$. Given any subset $A^{\prime} \subseteq A$, one can consider the partial identity $1_{A^{\prime}}$. The corresponding binary relation is $\left\{(a, a) \mid a \in A^{\prime}\right\}$. The case of interest for us will be binary relations on a complete lattice, but we will only be interested in those relations that preserve the lattice structure.

Definition 2.4 (Abstract flow). An abstract $L$-flow is an element of $\mathscr{C}\left(L^{2}\right)$. If $L$ is understood from the context, then we simply call an element of $\mathscr{C}\left(L^{2}\right)$ an abstract flow.

If $f$ is an abstract flow, then $\operatorname{Im} f$ is a meet-closed subset of $L^{2}$ and hence a binary relation on $L$ that we denote by $\bar{f}$. So to make clear our notational conventions: $f \in \mathscr{C}\left(L^{2}\right)$ denotes a closure operator on $L \times L$ and $\bar{f} \in B(L)$ stands for the corresponding binary relation $\operatorname{Im} f$. This leads us to the suggestive notation $\ell_{1} f \ell_{2}$ for the value of $f$ on $\left(\ell_{1}, \ell_{2}\right) \in L^{2}$. Since many of our flows come from automata, we also use the illustrative notation

$$
\ell_{1} \stackrel{f}{\rightarrow} \ell_{2} .
$$

The elements of $\bar{f}$ are called the stable pairs of $f$. We write $\ell_{1} \bar{f} \ell_{2}$ to indicate $\left(\ell_{1}, \ell_{2}\right)$ is stable for $f$; we also use the pictorial notation

$$
\ell_{1} \stackrel{\bar{f}}{\rightarrow} \ell_{2}
$$

to indicate $\left(\ell_{1}, \ell_{2}\right)$ is stable under $f$.

We now want to show that $\mathscr{C}\left(L^{2}\right)$ is a submonoid of $B(L)$ under the identification $f \mapsto \bar{f}$. More precisely, we identify $\mathscr{C}\left(L^{2}\right)$ with the set of those binary relations on $L$ that are meet-closed (as subsets of $L \times L$ ).

Proposition 2.5. $\mathscr{C}\left(L^{2}\right)$ is a submonoid of $B(L)$ under the identification $f \mapsto \bar{f}$. That is, a multiplication can be defined on $\mathscr{C}\left(L^{2}\right)$ by setting $f g$ to be the closure operator associated to $\bar{f} \bar{g}$, turning $\mathscr{C}\left(L^{2}\right)$ into a monoid.

Proof. It is clear that the set of stable pairs of the identity relation $I_{L}$ is meetclosed and so belongs to $\mathscr{C}\left(L^{2}\right)$ under our identification. We need to show that if $f, g \in \mathscr{C}\left(L^{2}\right)$, then the set of stable pairs of $\overline{f g}=\bar{f} \bar{g}$ is meet-closed. The empty meet is $(T, T)$, where $T$ is the top of $L$. Since the sets of stable pairs of $f$ and $g$ are meet-closed,

$$
T \stackrel{\bar{f}}{\rightarrow} T \stackrel{\bar{g}}{\rightarrow} T
$$

and so $T \stackrel{\overline{f g}}{\longrightarrow} T$. Suppose $\left\{\left(\ell_{\alpha}, \ell_{\alpha}^{\prime}\right)\right\}_{\alpha \in A}$ is a non-empty subset of the set of stable pairs of $\overline{f g}$. Then, for each $\alpha \in A$, there is an element $\ell_{\alpha}^{\prime \prime} \in L$ so that

$$
\ell_{\alpha} \stackrel{\bar{f}}{\rightarrow} \ell_{\alpha}^{\prime \prime} \stackrel{\bar{g}}{\rightarrow} \ell_{\alpha}^{\prime}
$$

Since the stable pairs of $f$ and $g$ are meet-closed, we then obtain

$$
\bigwedge_{\alpha \in A} \ell_{\alpha} \stackrel{\bar{f}}{\rightarrow} \bigwedge_{\alpha \in A} \ell_{\alpha}^{\prime \prime} \stackrel{\bar{g}}{\rightarrow} \bigwedge_{\alpha \in A} \ell_{\alpha}^{\prime}
$$


showing that

$$
\bigwedge_{\alpha \in A} \ell_{\alpha} \stackrel{\overline{f g}}{\longrightarrow} \bigwedge_{\alpha \in A} \ell_{\alpha}^{\prime}
$$

Since meets in $L^{2}$ are coordinate-wise, this completes the proof.

We remark that $I_{L}$ has stable pairs of the form $\{(\ell, \ell) \mid \ell \in L\}$. Hence

$$
\ell_{1} I_{L} \ell_{2}=\left(\ell_{1} \vee \ell_{2}, \ell_{1} \vee \ell_{2}\right)
$$

So the multiplicative identity $I_{L}$ of $\mathscr{C}\left(L^{2}\right)$ is not the identity closure operator (which has stable set $L^{2}$ and is the bottom of $\left.\mathscr{C}\left(L^{2}\right)\right)$.

We shall call $\mathscr{C}\left(L^{2}\right)$ the abstract flow monoid on $L$. From its description as a submonoid of $B(L)$ we immediately obtain:

Proposition 2.6. $\mathscr{C}\left(L^{2}\right)$ is an ordered monoid. That is, $f_{1} \leq f_{2}$ and $g_{1} \leq g_{2}$ imply $f_{1} g_{1} \leq f_{2} g_{2}$.

Remark 2.7. It is convenient to know which binary relations come from 2-variable closure operators from the point of view of relations on $L$ as maps $L \rightarrow P(L)$. We observe that $f \subseteq L \times L$ is meet-closed if and only if $\ell_{\alpha}^{\prime} \in \ell_{\alpha} f$, for $\alpha \in A$, implies $\bigwedge_{\alpha \in A} \ell_{\alpha}^{\prime} \in\left(\bigwedge_{\alpha \in A} \ell_{\alpha}\right) f$, where we view $f$ as a map $L \rightarrow P(L)$ for the moment. This is analogous to relational morphisms [9, 30] in the sense that the algebraic structure is preserved by the relation.

2.2.1. From one-variable to two-variable closure operators and back again. It turns out to be useful to identify $\mathscr{C}(L)$ with a certain submonoid of the abstract flow monoid $\mathscr{C}\left(L^{2}\right)$. This will allow one-variable operators to act on the left and right of abstract flows by inner translations.

Proposition 2.8. Let $f \in \mathscr{C}(L)$. Define $\varphi: \mathscr{C}(L) \rightarrow B(L)$ by $f \mapsto 1_{\operatorname{Im} f}$, where the latter partial identity is viewed as a binary relation. Then:

(1) $f \varphi \in \mathscr{C}\left(L^{2}\right)$;

(2) $\varphi$ is an order-embedding;

(3) the identity closure operator maps to the identity relation;

(4) $f \varphi g \varphi=(f \vee g) \varphi$;

(5) $\mathscr{C}(L) \varphi$ is an idempotent, commutative submonoid of $\mathscr{C}\left(L^{2}\right)$ isomorphic to $\mathscr{C}(L)$ with the join operation.

Proof. The first three items are trivial. For (4), $1_{\operatorname{Im} f} 1_{\operatorname{Im} g}=1_{\operatorname{Im} f \cap \operatorname{Im} g}=1_{\operatorname{Im} f \vee g}$. The final item is immediate from the previous one.

From now on we identify $f \in \mathscr{C}(L)$ with $f \varphi$ and drop the latter notation. To make things more concrete, if $f \in \mathscr{C}(L)$ is a closure operator on $L$, then the corresponding meet-closed binary relation is

$$
\bar{f}=\{(\ell, \ell) \mid \ell f=\ell\} .
$$

Proposition 2.8 allows us to view the join-lattice $\mathscr{C}(L)$ as operating on the left and right of $\mathscr{C}\left(L^{2}\right)$. The following proposition is immediate from the definitions and is stated merely for the convenience of the reader.

Proposition 2.9. Let $h \in \mathscr{C}(L)$ and $f \in \mathscr{C}\left(L^{2}\right)$. Then $\ell \stackrel{\overline{h f}}{\longrightarrow} \ell^{\prime}$ if and only if $\ell h=\ell$ and $\ell \stackrel{\bar{f}}{\rightarrow} \ell^{\prime}$. Dually, $\ell \stackrel{\overline{f h}}{\longrightarrow} \ell^{\prime}$ if and only if $\ell^{\prime} h=\ell^{\prime}$ and $\ell \stackrel{\bar{f}}{\rightarrow} \ell^{\prime}$. 
Viewing flows as binary relations, if $h \in \mathscr{C}(L)$ and $f \in \mathscr{C}\left(L^{2}\right)$, then $\overline{h f}$ is the binary relation obtained by restricting the domain of $\bar{f}$ to $\operatorname{Im} h$. Similarly, $\overline{f h}$ is the binary relation obtained by restricting the range of $\bar{f}$ to $\operatorname{Im} h$.

The following proposition establishes the basic properties of our action.

Proposition 2.10. Let $g, g_{1}, g_{2} \in \mathscr{C}(L)$ and $f, f^{\prime} \in \mathscr{C}\left(L^{2}\right)$.

(1) $g_{1} g_{2} f=g_{2} g_{1} f=\left(g_{1} \vee g_{2}\right) f$;

(2) $f g_{1} g_{2}=f g_{2} g_{1}=f\left(g_{1} \vee g_{2}\right)$;

(3) $1_{L} g=g=g 1_{L}$;

(4) $f \leq g_{1} f g_{2}$.

Proof. Proposition 2.8 immediately yields (1), (2) and (3). Item (4) follows from $1_{L}$ being the bottom of $\mathscr{C}(L)$ and Proposition 2.8 .

2.2.2. Back-flow, forward-flow and star. To any binary relation $f$ on $L$, we can associate three subsets of $L$ : the domain $\operatorname{dom} f$, the range $\operatorname{ran} f$ and the fixed point set fix $f$ (where fix $f=\{\ell \in L \mid(\ell, \ell) \in f\}$ ). It is immediate that if $\bar{f}$ is the relation associated to $f \in \mathscr{C}\left(L^{2}\right)$ (and so $\bar{f}$ is meet-closed), then these sets are meet-closed and hence define one-variable closure operators (which we can view as two-variable closure operators in our usual way).

Proposition 2.11. Let $f \in \mathscr{C}\left(L^{2}\right)$. Then $\operatorname{dom} \bar{f}$, ran $\bar{f}$ and fix $\bar{f}$ are meet-closed subsets of $L$ and hence correspond to one-variable closure operators.

Proof. Clearly, $T \in \operatorname{dom} \bar{f}$. If $\left\{\ell_{\alpha} \mid \alpha \in A\right\} \subseteq \operatorname{dom} \bar{f}$, then we can find, for all $\alpha \in A$, an element $\ell_{\alpha}^{\prime} \in L$ so that $\ell_{\alpha} \stackrel{\bar{f}}{\rightarrow} \ell_{\alpha}^{\prime}$. Then since $f$ is a closure operator on $L^{2}$, we have $\bigwedge_{\alpha \in A} \ell_{\alpha} \stackrel{\bar{f}}{\rightarrow} \bigwedge_{\alpha \in A} \ell_{\alpha}^{\prime}$ and so $\bigwedge_{\alpha \in A} \ell_{\alpha} \in \operatorname{dom} \bar{f}$. Similarly, ran $\bar{f}$ is meet-closed.

For the fixed point set, suppose $\ell_{\alpha} \stackrel{\bar{f}}{\rightarrow} \ell_{\alpha}$ for all $\alpha \in A$. Then

$$
\bigwedge_{\alpha \in A} \ell_{\alpha} \stackrel{\bar{f}}{\rightarrow} \bigwedge_{\alpha \in A} \ell_{\alpha},
$$

whence $\bigwedge_{\alpha \in A} \ell_{\alpha} \in$ fix $\bar{f}$, completing the proof.

Because we deal with deterministic automata (but not necessarily co-deterministic automata), we have little occasion to use $\operatorname{ran} \bar{f}$. Let us give names to the associated closure operators for the other two sets.

Definition 2.12 (Back-flow and Kleene star). Let $f \in \mathscr{C}\left(L^{2}\right)$. Then we define the following one-variable closure operators associated to $f$ :

(1) Define $\overleftarrow{f}$ to be the closure operator on $L$ with image $\operatorname{dom} \bar{f}$. It is called back-flow along $f$.

(2) Define $f^{*}$ to be the closure operator on $L$ with image fix $\bar{f}$. It is called the Kleene star of $f$.

The names will be motivated a little bit later when we look at the set-partition lattice and flows on automata. For instance, Remark 2.26 will motivate back-flow.

Since the operations of taking the domain and the fixed-point set of a relation are order-preserving, it follows that the maps $f \mapsto \overleftarrow{f}$ and $f \mapsto f^{*}$ are order-preserving. Also since fix $\bar{f} \subseteq \operatorname{dom} \bar{f}$, we have $\overleftarrow{f} \leq f^{*}$ 
It is sometimes convenient to work with a direct description of the back-flow

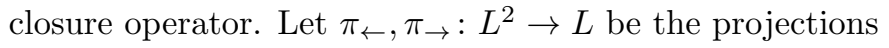

$$
\begin{aligned}
& \left(\ell, \ell^{\prime}\right) \pi_{\leftarrow}=\ell, \\
& \left(\ell, \ell^{\prime}\right) \pi_{\rightarrow}=\ell^{\prime} .
\end{aligned}
$$

The letters $b$ and $f$ stand for back and front (we are thinking in pictures $\ell \rightarrow \ell^{\prime}$ ). Notice that $\pi_{\rightarrow}, \pi_{\leftarrow}$ are complete lattice homomorphisms.

Proposition 2.13. Let $f \in \mathscr{C}\left(L^{2}\right)$ and $\ell \in L$. Then

$$
\overleftarrow{\ell f}=(\ell \stackrel{f}{\rightarrow} B) \pi_{\leftarrow}
$$

where, as usual, $B$ is the bottom of $L$.

Proof. By definition $\ell \overleftarrow{f}$ is the least element $\ell^{\prime}$ in the domain of $\bar{f}$ such that $\ell \leq \ell^{\prime}$ So suppose $\left(\ell^{\prime}, \ell^{\prime \prime}\right)$ is a stable pair for $f$ with $\ell \leq \ell^{\prime}$. Then $(\ell, B) \leq\left(\ell^{\prime}, \ell^{\prime \prime}\right)$ and so $(\ell \stackrel{f}{\rightarrow} B) \leq\left(\ell^{\prime}, \ell^{\prime \prime}\right)$. Thus the right hand side of $(2.3)$ is the minimal element of $\operatorname{dom} \bar{f}$ that is above $\ell$.

This proposition explains to some extent the terminology back-flow. Since $f$ is order-preserving, $\ell \overleftarrow{f} \leq\left(\ell f \ell^{\prime}\right) \pi_{\leftarrow}$ for any $\ell, \ell^{\prime} \in L$. So $\ell^{\overleftarrow{f}}$ is picking up whatever always flows back to $\ell$. We are also interested in what must flow forward.

Definition 2.14 (Forward-flow). If $f \in \mathscr{C}\left(L^{2}\right)$ and $\ell \in L$, we define an orderpreserving map $\vec{f}: L \rightarrow L$ by

$$
\vec{\ell}=(\ell \stackrel{f}{\rightarrow} B) \pi_{\rightarrow}
$$

where $B$ is the bottom of $L$. We call $\vec{f}$ the forward-flow along $f$.

That $\vec{f}$ is order-preserving follows as $\ell \leq \ell^{\prime}$ implies $(\ell, B) \leq\left(\ell^{\prime}, B\right)$. Notice that we use $\overleftarrow{f}$ for back-flow since the arrow points backwards and $\vec{f}$ for forward-flow for the opposite reason. See Remark 2.26] below for motivation.

Remark 2.15. If we view $f \in \mathscr{C}(L)$ as a two-variable closure operator, then $\operatorname{dom} \bar{f}=$ $\operatorname{Im} f$ and so $f=\overleftarrow{f}$. In fact, $\ell \stackrel{f}{\rightarrow} B=(\ell f, \ell f)$ and so $f=\vec{f}$, as well.

Proposition 2.16. The map $f \mapsto \vec{f}$ from $\mathscr{C}\left(L^{2}\right)$ to the monoid of order-preserving maps on $L$ satisfies, for $f, g \in \mathscr{C}\left(L^{2}\right)$,

$$
\vec{f} \vec{g} \leq \overrightarrow{f g},
$$

where the order is taken pointwise. It also sends the identity to the identity.

Proof. Since dom $I_{L}=L$, which in turn is the image of the identity closure operator on $L$, it follows that $\overleftarrow{I_{L}}$ is the identity map on $L$.

Suppose $f, g \in \mathscr{C}\left(L^{2}\right)$ and $\ell \in L$. If $\ell \stackrel{f g}{\longrightarrow} B=\left(\ell_{1}, \ell_{2}\right)$, then there exists $\ell^{\prime} \in L$ such that $\ell_{1} \stackrel{\bar{f}}{\rightarrow} \ell^{\prime} \stackrel{\bar{g}}{\rightarrow} \ell_{2}$. Since $\ell \leq \ell_{1}$ and $B \leq \ell^{\prime}$, it follows that $\ell \stackrel{f}{\rightarrow} B \leq\left(\ell_{1}, \ell^{\prime}\right)$ and so $\ell \vec{f} \leq \ell^{\prime}$. Because $B \leq \ell_{2}$, we have $\ell^{\prime} \vec{g} \leq \ell_{2}$. Thus

$$
\ell \vec{f} \vec{g} \leq \ell^{\prime} \vec{g} \leq \ell_{2}=\ell \overrightarrow{f g}
$$

as required. 
In general equality does not hold in (2.4). However, in the situation that will be of primary interest to us, it will turn out to hold. Namely, when there is no back-flow, equality holds as the following proposition demonstrates.

Proposition 2.17. Suppose $\ell \in L$ and $f, g \in \mathscr{C}\left(L^{2}\right)$ are such that $\ell \overleftarrow{f}=\ell$, $(\ell \vec{f}) \overleftarrow{g}=\ell \vec{f}$. Then

$$
\ell \vec{f} \vec{g}=\ell \overrightarrow{f g}
$$

Proof. By Proposition 2.16, it suffices to prove that $\ell \overrightarrow{f g} \leq \ell \vec{f} \vec{g}$. Let $\ell^{\prime}=\ell \vec{f}$. By hypothesis on $\ell$ and $f, \ell \stackrel{f}{\rightarrow} B=\left(\ell, \ell^{\prime}\right)$. By hypothesis on $\ell^{\prime}$ and $g, \ell^{\prime} \stackrel{g}{\rightarrow} B=$ $\left(\ell^{\prime}, \ell^{\prime} \vec{g}\right)$. Thus $\ell \stackrel{\overline{f g}}{\longrightarrow} \ell^{\prime} \vec{g}$. Hence

$$
\ell \stackrel{f g}{\longrightarrow} B \leq \ell \stackrel{f g}{\rightarrow} \ell^{\prime} \vec{g}=\left(\ell, \ell^{\prime} \vec{g}\right)
$$

establishing $\ell \overrightarrow{f g} \leq \ell \vec{f} \vec{g}$, as required.

2.3. Flow lattices. For this section, fix a finite non-empty alphabet $X$. We shall need the notion of an $X$-flow lattice. Examples of flow lattices arise from trying to compute complexity via the Presentation Lemma [6, 35, 30, as well as when trying to compute pointlikes for certain pseudovarieties [10, 12. If $X$ is a set, $X^{*}$ denotes the free monoid generated by $X$.

Definition 2.18 (Flow lattice). An $X$-flow lattice is a complete lattice $L$ equipped with a map $\Phi: X \rightarrow \mathscr{C}\left(L^{2}\right)$ or equivalently, abusing notation, a homomorphism $\Phi: X^{*} \rightarrow \mathscr{C}\left(L^{2}\right)$. The closure operator $w \Phi$, for $w \in X^{*}$, is called free flow along $w$ and we denote it in arrow notation by $\stackrel{w}{\rightarrow}$.

Let us give a motivating example. Fix for the rest of the paper an $X$-generated finite group mapping monoid $M[17,30$. That is, $M$ has a 0 -minimal regular ideal $I$ (necessarily unique), containing a non-trivial group, such that $M$ acts faithfully on both the left and right of $I$. Also fix an $\mathscr{R}$-class $R$ of $I$, which shall be termed the distinguished $\mathscr{R}$-class of $M$. We view $(R, M)$ as a faithful partial transformation monoid [30, Chapter 4].

Definition 2.19 (Set flow lattice). Take $\mathrm{S}(M, X)=(P(R), \subseteq)$. This is called the set flow lattice for $M$. To make $\mathrm{S}(M, X)$ into an $X$-flow lattice, define $x \Phi$ by $X \stackrel{\bar{x}}{\rightarrow} Y$ if and only if $X x \subseteq Y$.

In this paper, we use the convention that if $(Q, M)$ is a partial transformation monoid or automaton and $q m$ is not defined, then we write $q m=\emptyset$.

Proposition 2.20. $\mathrm{S}(M, X)$ is an $X$-flow lattice.

Proof. The top of $\mathrm{S}(M, X)$ is $R$ and clearly $R x \subseteq R$, so the set of stable pairs of $x \Phi$ is closed under empty meets. If $U x \subseteq Y$ and $Z x \subseteq W$, then clearly $(U \cap Z) x \subseteq$ $Y \cap W$. So $\overline{x \Phi}$ is closed under finite meets.

We remark that the set flow lattice does not depend on the choice of $R$ since all $\mathscr{R}$-classes are isomorphic via left multiplication. Another important example is 
the set-partition flow lattice of $M$. In this paper, we do not distinguish between a partition and its associated equivalence relation.

Definition 2.21 (Set-partition flow lattice). The set-partition flow lattice $\operatorname{SP}(M, X)$ consists of all pairs $(Y, P)$, where $Y \subseteq R$ and $P$ is a partition on $Y$. This is a lattice where $(Y, P) \leq(Z, Q)$ if and only if $Y \subseteq Z$ and $y P y^{\prime}$ implies y $Q y^{\prime}$; in other words, the inclusion of $Y$ into $Z$ induces a well-defined map $Y / P \rightarrow Z / Q$.

It is easily verified that $\operatorname{SP}(M, X)$ is a lattice. The top is given by $(R,\{R\})$, that is, the set $R$ with a single block for the partition. The bottom is $(\emptyset, \emptyset)$. The meet is given by

$$
(U, P) \wedge(Y, Q)=(U \cap Y, P \wedge Q),
$$

where the blocks of $P \wedge Q$ consist of all non-empty intersections of the form $B \cap B^{\prime}$ with $B$ a block of $P$ and $B^{\prime}$ a block of $Q$. The join is easily verified to be given by

$$
(U, P) \vee(Y, Q)=(U \cup Y, P \vee Q),
$$

where $P \vee Q$ is the transitive closure of $P \cup Q$ viewed as a relation on $U \cup Y$, that is, the equivalence relation on $U \cup Y$ generated by $P$ and $Q$. Again there is no dependence on the choice of the $\mathscr{R}$-class $R$ in the definition of $\operatorname{SP}(M, X)$.

The set-partition flow lattice is used in computing complexity via the Presentation Lemma [6, 35. More details will be given in the next section.

To make $\operatorname{SP}(M, X)$ an $X$-flow lattice, we declare

$$
(U, P) \stackrel{\bar{x}}{\rightarrow}(Y, Q)
$$

if and only if $U x \subseteq Y$ and the partial function $\cdot x: U \rightarrow Y$ induces a well-defined partial injective map $\cdot x: U / P \rightarrow Y / Q$. This means that if $m, n \in U$ and $m x, n x \in R$ (and hence in $Y$ ), then

$$
m P n \Longleftrightarrow m x Q n x .
$$

In this way we have defined $x \Phi$.

Proposition 2.22. $\mathrm{SP}(M, X)$ is an $X$-flow lattice.

Proof. Clearly $(R,\{R\}) \stackrel{\bar{x}}{\rightarrow}(R,\{R\})$. Suppose now that

$$
\left(U_{1}, P_{1}\right) \stackrel{\bar{x}}{\rightarrow}\left(Y_{1}, Q_{1}\right) \text { and }\left(U_{2}, P_{2}\right) \stackrel{\bar{x}}{\rightarrow}\left(Y_{2}, Q_{2}\right) .
$$

Then, as we saw above,

$$
\left(U_{1} \cap U_{2}\right) x \subseteq Y_{1} \cap Y_{2},
$$

so it suffices to show that

$$
\left(U_{1} \cap U_{2}\right) /\left(P_{1} \wedge P_{2}\right) \rightarrow\left(Y_{1} \cap Y_{2}\right) /\left(Q_{1} \wedge Q_{2}\right)
$$

is a partial injective function. Let $m, n \in U_{1} \cap U_{2}$ and suppose $x \in X$ is such that $m x, n x \in R$. Then $m P_{1} \wedge P_{2} n$ if and only if $m P_{1} n$ and $m P_{2} n$. But by (2.5) this occurs if and only if $m x Q_{1} n x$ and $m x Q_{2} n x$, that is, if and only if $m x Q_{1} \wedge Q_{2} n x$. This completes the proof that the set of stable pairs of $\stackrel{x}{\rightarrow}$ is meet-closed.

Notice that there is a natural lattice homomorphism from $\mathrm{SP}(M, X)$ to $\mathrm{S}(M, X)$ preserving the $X$-flow lattice structure. Here by a lattice homomorphism, we mean a map preserving both meets and joins.

It is worth describing the closure operator $w \Phi$ for strings $w \in X^{*}$. 
Proposition 2.23. Let $w \in X^{*}$. Then $(Y, P) \stackrel{\bar{w}}{\rightarrow}(Z, Q)$ if and only if $Y w \subseteq Z$ and $r P s \Longleftrightarrow r w Q$ sw for all $r, s \in Y$ with $r w, s w \in R$.

Proof. Let $w=x_{1} \cdots x_{n}$ with the $x_{i} \in X$. First assume $(Y, P) \stackrel{\bar{w}}{\rightarrow}(Z, Q)$. Then we can find $\left(Y_{1}, P_{1}\right), \ldots,\left(Y_{n-1}, P_{n-1}\right)$ so that

$$
(Y, P) \stackrel{\bar{x}_{1}}{\longrightarrow}\left(Y_{1}, P_{1}\right) \longrightarrow \cdots \longrightarrow\left(Y_{n-1}, P_{n-1}\right) \stackrel{\bar{x}_{n}}{\longrightarrow}(Z, Q) .
$$

Then $Y x_{1} \subseteq Y_{1}, Y_{1} x_{2} \subseteq Y_{2}, \ldots, Y_{n-1} x_{n} \subseteq Z$. Thus $Y w \subseteq Z$. Also right multiplication by $w$ induces a partial injective map $Y / P \rightarrow Z / Q$, namely the composition of partial injective maps

$$
Y / P \stackrel{\cdot x_{1}}{\longrightarrow} Y_{1} / P_{1} \longrightarrow \cdots \longrightarrow Y_{n-1} / P_{n-1} \stackrel{\cdot x_{n}}{\longrightarrow} Z / Q
$$

Conversely, suppose the conditions of the proposition hold. Set $Y_{0}=Y, Y_{n}=Z$ and $Y_{i+1}=Y_{i} x_{i+1}$ for $0 \leq i \leq n-2$. From $Y w \subseteq Z$, it follows that $Y_{n-1} x_{n} \subseteq Z$. Assume inductively that we have a partition $P_{i}$ on $Y_{i}$, for $0 \leq i \leq n-2$, so that $\left(Y_{i-1}, P_{i-1}\right) \stackrel{\bar{x}_{i}}{\longrightarrow}\left(Y_{i}, P_{i}\right)$ and

$$
r x_{i+1} \cdots x_{n} Q s x_{i+1} \cdots x_{n} \Longleftrightarrow r P_{i} s
$$

for $r, s \in Y_{i}$ and $r x_{i+1} \cdots x_{n}, s x_{i+1} \cdots x_{n} \in R$. The base case is the hypothesis (take $\left.P_{0}=P\right)$. For the general case, noting that $Y_{i+1}=Y_{i} x_{i+1}$, set $r x_{i+1} P_{i+1} s x_{i+1}$ if and only if $r P_{i} s$. This is well defined because if $r x_{i+1}=r^{\prime} x_{i+1}$, then $r x_{i+1} \cdots x_{n}=$ $r^{\prime} x_{i+1} \cdots x_{n}$ and hence $r P_{i} r^{\prime}$ by hypothesis. It is then immediate from the construction that $\left(Y_{i}, P_{i}\right) \stackrel{\bar{x}_{i+1}}{\longrightarrow}\left(Y_{i+1}, P_{i+1}\right)$. Suppose $r x_{i+2} \cdots x_{n}, s x_{i+2} \cdots x_{n} \in R$ for $r, s \in Y_{i+1}$. Write $r=r^{\prime} x_{i+1}, s=s^{\prime} x_{i+1}$ with $r^{\prime}, s^{\prime} \in Y_{i}$. Then $r P_{i+1} s$ if and only if $r^{\prime} P_{i} s^{\prime}$, if and only if $r^{\prime} x_{i+1} \cdots x_{n} Q s^{\prime} x_{i+1} \cdots x_{n}$, if and only if $r x_{i+2} \cdots x_{n} Q s x_{i+2} \cdots x_{n}$. This completes the induction. By construction, we have

$$
(Y, P) \stackrel{\bar{x}_{1}}{\longrightarrow}\left(Y_{1}, P_{1}\right) \longrightarrow \cdots \longrightarrow\left(Y_{n-1}, P_{n-1}\right) \stackrel{\bar{x}_{n}}{\longrightarrow}(Z, Q)
$$

and so $(Y, P) \stackrel{\bar{w}}{\longrightarrow}(Z, Q)$, as required.

Definition 2.24 (Points). By a point of $\mathrm{S}(M, X)$ we mean an element of $R$ (viewed as a singleton). By a point of $\mathrm{SP}(M, X)$ we main a pair $(\{r\},\{\{r\}\})$, which we denote simply by $(r, r)$.

A key property of points in either of the above two settings is that if $p$ is a point and $x \in X$, then $p \stackrel{x}{\rightarrow} B=(p, q)$, where $q$ is either the bottom or a point. More precisely, we have the following statement, which is immediate from the definitions.

Proposition 2.25. Let $(r, r)$ be a point of $\mathrm{SP}(M, X)$ and let $x \in X$. Then

$$
(r, r) \stackrel{x}{\rightarrow} B= \begin{cases}(r x, r x) & r x \in R, \\ B & \text { else. }\end{cases}
$$

We are now in a position to explain the terminology forward-flow and back-flow.

Remark 2.26 (Explanation of back-flow). First consider the set flow lattice $\mathrm{S}(M, X)$. Then it is easy to see that $U \stackrel{x}{\rightarrow} B=(U, U x)$ for $x \in X$ and $U \subseteq R$. Thus $U \overleftarrow{x}=U$ and $U \vec{x}=U x$. That is, sets only flow forward. On the other hand, back-flow can occur for the set-partition flow lattice. For example, suppose that $m, n \in U, x \in X$ 
and $m x=n x \in R$. Assume further that $m$ and $n$ are in different blocks of the partition $P$. Then

$$
((U, P) \stackrel{x}{\rightarrow}(\emptyset, \emptyset)) \pi_{\leftarrow}
$$

will be of the form $\left(U, P^{\prime}\right)$, where in $P^{\prime}$ the blocks of $m$ and $n$ are joined together (and maybe more). Thus when one flows along $x$, there is some information flowing backwards.

The following important proposition gives a better understanding of back-flow and forward-flow.

Proposition 2.27. Let $w \in X^{*}$ and suppose that $(Y, P) \overleftarrow{w}=(Y, P)$. Let $P=$ $\left\{B_{1}, \ldots, B_{r}\right\}$, where $B_{1}, \ldots, B_{k}$ are the blocks of $P$ with $B_{i} w \neq \emptyset$. Then $B_{1} w, \ldots$, $B_{k} w$ are disjoint and $(Y, P) \vec{w}=\left(Y w,\left\{B_{1} w, \ldots, B_{k} w\right\}\right)$.

Proof. Let $(Y, P) \vec{w}=(Z, Q)$. Then $(Y, P) \stackrel{w}{\rightarrow}(\emptyset, \emptyset)=((Y, P),(Z, Q))$ by hypothesis. By Proposition 2.23 , it follows that $Y w \subseteq Z$ and $Y / P \stackrel{\cdot w}{\longrightarrow} Z / Q$ is a partial injective map. Consequently, $B_{i} w \cap B_{j} w \neq \emptyset$ implies $i=j$. Setting $P^{\prime}=\left\{B_{1} w, \ldots, B_{k} w\right\}$, we have $\left(Y w, P^{\prime}\right) \in \mathrm{SP}(M, X)$ and $\left(Y w, P^{\prime}\right) \leq(Z, Q)$. Moreover, $(Y, P) \stackrel{\bar{w}}{\longrightarrow}\left(Y w, P^{\prime}\right)$ since $Y / P \stackrel{\cdot w}{\longrightarrow} Y w / P^{\prime}$ is trivially an injective partial map. Thus $(Z, Q)=\left(Y w, P^{\prime}\right)$, as required.

There is a straightforward generalization of these flow lattices to any $X$-generated faithful partial transformation monoid $(Q, M)$.

2.4. Flows on automata. One can build new closure operators on $L^{2}$ via composition and Kleene star. In fact, there is a convenient formalism, via automata, to construct more elaborate closure operators. Given an $X$-flow lattice, one has an immediate interpretation of any automaton over the alphabet $X$ as a closure operator. This motivates our arrow notation and the use of the Kleene star.

Fix a lattice $L$. By an $L$-automaton $\mathscr{A}=(Q, \delta)$ we mean a finite directed graph with vertex set $Q$ whose edge set $E$ is labelled by elements of $\mathscr{C}\left(L^{2}\right)$ via $\delta: E \rightarrow$ $\mathscr{C}\left(L^{2}\right)$. We continue to fix a finite alphabet $X$. Suppose, in addition, that $L$ is an $X$-flow lattice and $\mathscr{A}=(Q, X)$ is an automaton (possibly non-deterministic) 8] over $X$ (we say an $X$-automaton). Here $Q$ denotes the state set, while the transition function is assumed to be understood. All automata are assumed finite. By abusing the distinction between elements $x \in X$ and the associated free flow $x \Phi$ operator we may view $\mathscr{A}$ as an $L$-automaton. If $\mathscr{A}$ is a partial deterministic automaton (that is, $X$ acts on $Q$ by partial functions $[8]$ ), then the completion $\mathscr{A}^{\square}$ of $\mathscr{A}$ is obtained by adding a sink state $\square[8$. Often we will write "partial automaton" as an abbreviation for partial deterministic automaton.

By convention, if we draw a finite graph with the vertices labelled by lattice elements from $L$ and the edges labelled by various $\bar{f}$ with $f \in \mathscr{C}\left(L^{2}\right)$, then we assume the lattice elements labelling the initial and terminal vertices of each edge $e$ form a stable pair for the closure operator labelling $e$.

We remark that many of our definitions make sense for any lattice $L$. Only when speaking about elements of $X$ do we need to consider $X$-flow lattices.

Definition 2.28 (Flow on an $L$-automaton). Let $\mathscr{A}=(Q, \delta)$ be an $L$-automaton. By an $L$-flow on $\mathscr{A}$, or just a flow if $L$ is understood, we mean a function $F: Q \rightarrow L$ satisfying $q F \stackrel{\bar{e} \delta}{\longrightarrow} q^{\prime} F$ for each edge $q \stackrel{e}{\rightarrow} q^{\prime}$ of $\mathscr{A}$. 
We need to consider complete set-partition flows for the case of $\mathrm{SP}(M, X)$.

Definition 2.29 (Complete flow on an automaton). An $\operatorname{SP}(M, X)$-flow $F$ on a partial deterministic $X$-automaton $(Q, X)$ is called a complete flow if:

(1) $F$ extends to an $\operatorname{SP}(M, X)$-flow on $\mathscr{A}^{\square}$ via $\square F=B$;

(2) $F$ is fully defined, meaning, for each $r \in R$, there is a state $q \in Q$ such that $(r, r) \leq q F$.

Conditions (1) and (2) are to guarantee that $F$ comes from a relational morphism as we shall see in Section 3 ,

For example, if $x \in X$, consider the partial automaton $\mathscr{A}[x]$ given by

$$
q_{0} \stackrel{x}{\rightarrow} q_{1} .
$$

Then $F:\left\{q_{0}, q_{1}\right\} \rightarrow L$ is a flow if and only if $q_{0} F \stackrel{\bar{x}}{\rightarrow} q_{1} F$; that is, flows on $\mathscr{A}[x]$ correspond to stable pairs for free flow along $x$.

Let $\mathscr{A}=(Q, \delta)$ be an $L$-automaton. The set of flows on $\mathscr{A}$ is denoted $F L(\mathscr{A})$. If $L=\mathrm{SP}(M, X)$ and $\mathscr{A}$ is a partial deterministic $X$-automaton, the set of complete flows on $\mathscr{A}$ is denoted $C F L(\mathscr{A})$. We can view $F L(\mathscr{A})$ and $C F L(\mathscr{A})$ as subsets of the complete lattice $L^{Q}$ with coordinate-wise operations.

Proposition 2.30. Let $\mathscr{A}=(Q, \delta)$ be an L-automaton. The set $F L(\mathscr{A})$ is a meet-closed subset of $L^{Q}$.

Proof. If $q F=T$ for all $q \in Q$, then $F$ is a flow since $T \stackrel{\overline{e \delta}}{\longrightarrow} T$ for all edges $e$. This yields closure under empty meets. Let $\left\{F_{\alpha}: Q \rightarrow L \mid \alpha \in A\right\}$ be a collection of flows. Then, for each edge $q \stackrel{e}{\rightarrow} q^{\prime}$, we have $q F_{\alpha} \stackrel{\overline{e \delta}}{\longrightarrow} q^{\prime} F_{\alpha}$ for all $\alpha \in A$. Since $\overline{e \delta}$ is meet-closed, we see that

$$
\bigwedge_{\alpha \in A} q F_{\alpha} \stackrel{\overline{e \delta}}{\longrightarrow} \bigwedge_{\alpha \in A} q^{\prime} F_{\alpha}
$$

and so $\bigwedge_{\alpha \in A} F_{\alpha}$ is a flow.

It follows that $F L(\mathscr{A})$ gives rise to a closure operator on $L^{Q}$. That is, if we label each vertex of $\mathscr{A}$ by an element of $L$ via a function $f: Q \rightarrow L$, then there is a least flow $\overline{\mathscr{A}}(f): Q \rightarrow L$ on $\mathscr{A}$ such that $q f \leq q \overline{\mathscr{A}}(f)$ for all $q \in Q$. In general, $C F L(\mathscr{A})$ is not meet-closed. In fact, it almost never contains the empty meet.

Example 2.31. Let us consider an example of a flow with $L$ the set flow lattice. Suppose we have a pointed complete automaton $\mathscr{A}=(Q, X)$ with a base point $q_{0}$ such that $q_{0} X^{*}=Q$. Let $N$ be the transition monoid of $\mathscr{A}$. Fix a base point $r_{0} \in R$. Consider the smallest relational morphism [9] $\varphi:(R, M) \rightarrow(Q, N)$ such that $r_{0}$ relates $q_{0}$ and such that if $m \in R, m x \in R$ and $m$ relates to $q$, then $m x$ relates to $q x$. That is,

$$
r \varphi=\left\{q_{0} \cdot w \mid w \in X^{*} \text { and } r=r_{0} \cdot w\right\} .
$$

This is a fully-defined relation since $\mathscr{A}$ is complete. Define a function $f: Q \rightarrow P(R)$ that assigns $\left\{r_{0}\right\}$ to $q_{0}$ and $\emptyset$ to every other vertex. Then $q \overline{\mathscr{A}}(f)=q \varphi^{-1}$.

In the same context, if we use the set-partition flow lattice, then in addition to computing the relational morphism $\varphi$, we will be computing the partitions giving 
rise to the minimal injective automaton congruence on the derived transformation semigroup 9] (viewed as an automaton) of $\varphi$. More details will follow in the next section.

If $\mathscr{A}=(Q, \delta)$ is an $L$-automaton and $q \neq q^{\prime} \in Q$, we can obtain a new abstract flow on $L$ by sampling at the states $q, q^{\prime}$.

Definition 2.32 (Sampling at two states). Let $\mathscr{A}=(Q, \delta)$ be an $L$-automaton and $q \neq q^{\prime} \in Q$. The abstract flow $\mathscr{A}\left(q, q^{\prime}\right) \in \mathscr{C}\left(L^{2}\right)$ is defined as follows. Let $\ell, \ell^{\prime} \in L$. Define $f: Q \rightarrow L$ by $q f=\ell, q^{\prime} f=\ell^{\prime}$ and by sending all other states to the bottom $B$ of $L$. Then we define

$$
\ell \stackrel{\mathscr{A}\left(q, q^{\prime}\right)}{\longrightarrow} \ell^{\prime}=\left(q \overline{\mathscr{A}}(f), q^{\prime} \overline{\mathscr{A}}(f)\right)
$$

to be the result of sampling $\mathscr{A}$ at $q, q^{\prime}$.

In other words, we consider all flows that are greater than or equal to $\ell$ at $q$ and to $\ell^{\prime}$ at $q^{\prime}$, take their meet and then sample the values at $q$ and $q^{\prime}$.

Proposition 2.33. $\mathscr{A}\left(q, q^{\prime}\right)$ is a closure operator. Moreover, if $\ell, \ell^{\prime} \in L$, then $\ell \stackrel{\overline{\mathscr{A}\left(q, q^{\prime}\right)}}{\longrightarrow} \ell^{\prime}$ if and only if there is a flow $F$ on $\mathscr{A}$ such that $q F=\ell$ and $q^{\prime} F=\ell^{\prime}$.

Proof. It is straightforward to verify that $\mathscr{A}\left(q, q^{\prime}\right)$ is order-preserving and increasing. To see that it is idempotent, suppose that $\ell_{1} \mathscr{A}\left(q, q^{\prime}\right) \ell_{2}=\left(\ell_{1}^{\prime}, \ell_{2}^{\prime}\right)$. Define $f: Q \rightarrow L$ by $q f=\ell_{1}, q^{\prime} f=\ell_{2}$ and by sending all other states to the bottom $B$ of $L$; let $f^{\prime}$ be defined analogously but with $q f^{\prime}=\ell_{1}^{\prime}$ and $q^{\prime} f^{\prime}=\ell_{2}^{\prime}$. Then $f \leq f^{\prime} \leq \overline{\mathscr{A}}(f)$ and so $\overline{\mathscr{A}}(f)=\overline{\mathscr{A}}\left(f^{\prime}\right)$ and hence $\ell_{1}^{\prime} \mathscr{A}\left(q, q^{\prime}\right) \ell_{2}^{\prime}=\left(\ell_{1}^{\prime}, \ell_{2}^{\prime}\right)$. Thus $\mathscr{A}\left(q, q^{\prime}\right)$ is a closure operator.

We next prove the second statement, describing the image $\overline{\mathscr{A}\left(q, q^{\prime}\right)}$ of $\mathscr{A}\left(q, q^{\prime}\right)$. Let $\ell, \ell^{\prime} \in L$ and define the map $f: Q \rightarrow L$ as in Definition 2.32, By definition of $\mathscr{A}\left(q, q^{\prime}\right)$, if $\ell \stackrel{\overline{\mathscr{A}\left(q, q^{\prime}\right)}}{\longrightarrow} \ell^{\prime}$, then

$$
\begin{aligned}
\ell & =q \overline{\mathscr{A}}(f), \\
\ell^{\prime} & =q^{\prime} \overline{\mathscr{A}}(f) .
\end{aligned}
$$

Conversely, if $F$ is a flow with $q F=\ell, q^{\prime} F=\ell^{\prime}$, then $f \leq F$ and therefore $\overline{\mathscr{A}}(f) \leq F$. Thus

$$
\begin{aligned}
\ell & =q f \leq q \overline{\mathscr{A}}(f) \leq q F=\ell, \\
\ell^{\prime} & =q^{\prime} f \leq q^{\prime} \overline{\mathscr{A}}(f) \leq q^{\prime} F=\ell^{\prime},
\end{aligned}
$$

establishing the second statement.

As an example, let $x \in X$ and consider the automaton

$$
\mathscr{A}[x]=q_{0} \stackrel{x}{\rightarrow} q_{1} .
$$

It follows directly from the definition that the closure operator $\mathscr{A}[x]\left(q_{0}, q_{1}\right)$ is free flow along $x$ (that is, the operator $\stackrel{x}{\rightarrow}$ ). Let us generalize this to free flow along a word $w \in X^{*}$. Suppose $w=x_{1} \cdots x_{n}$ and let

$$
\mathscr{A}[w]=q_{0} \stackrel{x_{1}}{\longrightarrow} q_{1} \stackrel{x_{2}}{\longrightarrow} \cdots \stackrel{x_{n}}{\longrightarrow} q_{n}
$$

Then the closure operator $\mathscr{A}[w]\left(q_{0}, q_{n}\right)$ is free flow along $w$. This follows from the following more general result. 
Proposition 2.34. Let $f_{1}, \cdots, f_{n} \in \mathscr{C}\left(L^{2}\right)$ and $f=f_{1} \cdots f_{n}$. Consider the $L$ automaton

$$
\mathscr{A}\left[f_{1}, \ldots, f_{n}\right]=q_{0} \stackrel{f_{1}}{\longrightarrow} q_{1} \stackrel{f_{2}}{\longrightarrow} \cdots \stackrel{f_{n}}{\longrightarrow} q_{n} .
$$

Then $\mathscr{A}\left[f_{1}, \ldots, f_{n}\right]\left(q_{0}, q_{n}\right)=f$.

Proof. Observe that, by Proposition $2.33, \ell \stackrel{\overline{\mathscr{A}\left[f_{1}, \ldots, f_{n}\right]\left(q_{0}, q_{n}\right)}}{\longrightarrow} \ell^{\prime}$ if and only if there is a flow $F$ on $\mathscr{A}\left[f_{1}, \ldots, f_{n}\right]$ with $q_{0} F=\ell$ and $q_{n} F=\ell^{\prime}$. This occurs if and only if we can choose a function $F: Q \rightarrow L$ so that $q_{0} F=\ell, q_{n} F=\ell^{\prime}$ and

$$
q_{i-1} F \stackrel{\bar{f}_{i}}{\longrightarrow} q_{i} F
$$

for all $i$. But since $f$ is the product $f_{1} \cdots f_{n}$, it follows that such a function $F$ exists if and only if $\ell \stackrel{\bar{f}}{\rightarrow} \ell^{\prime}$.

As another example, let $M$ and $N$ be $X$-generated monoids and put $L=P(M)$. Define an $X$-flow lattice structure on $L$ by $U \stackrel{\bar{x}}{\rightarrow} Y$ if and only if $U x \subseteq Y$. Let $\mathscr{A}$ be the right Cayley graph of $N$ with respect to $X$. Let $n \in N$. Then, for $1 \neq n \in N$,

$$
\{1\} \stackrel{\mathscr{A}(1, n)}{\longrightarrow} \emptyset=\left(1 \varphi^{-1}, n \varphi^{-1}\right),
$$

where $\varphi: M \rightarrow N$ is the canonical relational morphism respecting the generators $X$, that is, the relational morphism whose graph is the submonoid generated by the image of the diagonal map $X \rightarrow M \times N$.

We can also get a one-variable closure operator by sampling at a state.

Definition 2.35 (Sampling at a state). Let $\mathscr{A}=(Q, \delta)$ be an $L$-automaton and $q \in Q$. Then an element $\mathscr{A}(q) \in \mathscr{C}(L)$ can be defined as follows. Let $\ell \in L$. Define $f: Q \rightarrow L$ by $q f=\ell$ and by sending all other states to $B$. Then we define $\ell \mathscr{A}(q)=q \overline{\mathscr{A}}(f)$.

One can verify that $\mathscr{A}(q)$ is a closure operator in a similar fashion to Proposition 2.33 Many of our one-variable closure operators can be interpreted via sampling, as the following proposition, whose proof is merely unwinding the definitions, shows.

Proposition 2.36. Let $f \in \mathscr{C}\left(L^{2}\right)$. Then:

(1) $\overleftarrow{f}=\mathscr{A}[f]\left(q_{0}\right)$, where $\mathscr{A}[f]=q_{0} \stackrel{f}{\rightarrow} q_{1}$;

(2) $f^{*}=\mathscr{A}\left[f^{*}\right](q)$, where $\mathscr{A}\left[f^{*}\right]=q \bigcap^{f}$.

This proposition should explain the intuition behind the names back-flow and the Kleene star. Let's give further motivation for the star notation via an example. Let $w \in X^{*}$. Let $M$ be a finite $X$-generated group mapping monoid with distinguished $\mathscr{R}$-class $R$ and consider the set flow lattice on $R$. Let $U \in P(R)$. We claim that, for $w \in X^{*}$,

$$
U^{w}=U w^{*}
$$

where $w^{*}$ is the submonoid generated by the image of $w$ in $M$. Indeed,

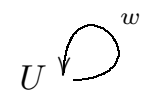


is, by definition, the least subset $Y$ containing $U$ such that $Y w \subseteq Y$. But this is exactly $U w^{*}$. Intuitively, the one-variable operator

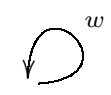

is obtained by taking the automaton $\mathscr{A}[w]$, identifying $q_{0}$ with $q_{n}$ and then sampling at $q_{0}$. Notice that the language of the resulting automaton is $w^{*}$.

If $f, g \in \mathscr{C}\left(L^{2}\right)$, we can define an $L$-automaton

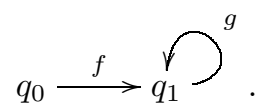

The two-variable closure operator obtained by sampling with respect to $\left(q_{0}, q_{1}\right)$ is none other than $f g^{*}$. Unwinding the definition we see that, for $\ell, \ell^{\prime} \in L$, we have $\ell \overline{f g^{*}} \ell^{\prime}$, drawn

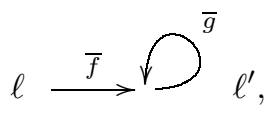

if and only if $\ell \stackrel{\bar{f}}{\rightarrow} \ell^{\prime}$ and $\ell^{\prime} \stackrel{\bar{g}}{\rightarrow} \ell^{\prime}$. The picture indicates that you flow from $\ell$ to $\ell^{\prime}$ via $f$ and then flow in a loop from $\ell^{\prime}$ to $\ell^{\prime}$ along $g$.

For instance, consider set flows on a finite $X$-generated group mapping monoid $M$ with distinguished $\mathscr{R}$-class $R$. Let $U, Y \subseteq R$ and $x, y \in X$. Then $U \stackrel{x y^{*}}{\longrightarrow} Y=$ $\left(U,(U x \cup Y) y^{*}\right)$.

Proposition 2.37. Let $f, g \in \mathscr{C}\left(L^{2}\right)$. Then:

(1) $f \leq f \overleftarrow{g} \leq f g^{*}$

(2) for all $k \geq 0, g^{k} \leq \bigvee_{m \geq 0} g^{m} \leq g^{*}$;

(3) $f \leq \bigvee_{k \geq 0}\left(f g^{k}\right) \leq f\left(\bigvee_{k \geq 0} g^{k}\right) \leq f g^{*}$;

(4) the map $g \mapsto g^{*}$ is a closure operator on $\mathscr{C}\left(L^{2}\right)$ and in particular $\left(g^{*}\right)^{*}=g^{*}$;

(5) $(f \vee g)^{*}=f^{*} g^{*}=f^{*} \vee g^{*}=g^{*} f^{*}$;

(6) $\left(f g^{*}\right)^{*}=f^{*} \vee g^{*}=\left(f^{*} g\right)^{*}$.

Proof. Proposition 2.10 establishes the first inequality of (1). The second follows since $\overleftarrow{g} \leq g^{*}$. The first inequality of (2) is clear. For the second, a stable pair for $g^{*}$ looks like $(\ell, \ell)$, where $\ell \stackrel{\bar{g}}{\rightarrow} \ell$. But then $\ell \stackrel{\overline{g^{m}}}{\longrightarrow} \ell$ for all $m \geq 0$ and hence $(\ell, \ell)$ is a stable pair for $\bigvee_{m \geq 0} g^{m}$. Item (3) is an immediate consequence of (2) and the fact that $\mathscr{C}\left(L^{2}\right)$ is an ordered monoid. The fourth item is trivial.

For (5), note that $1_{\text {fix } \bar{f}} 1_{\text {fix } \bar{g}}=1_{\text {fix } \bar{f} \cap \text { fix } \bar{g}}=1_{\text {fix } \overline{f \vee g}}$ and so $f^{*} g^{*}=(f \vee g)^{*}$. The remaining equalities follow from Proposition 2.8 Finally, for (6) we have by the previous parts that

$$
\left(f g^{*}\right)^{*} \leq\left(f^{*} g^{*}\right)^{*}=\left((f \vee g)^{*}\right)^{*}=(f \vee g)^{*}=f^{*} \vee g^{*} .
$$

Conversely, suppose that $\ell \in$ fix $\overline{f g^{*}}$. Then

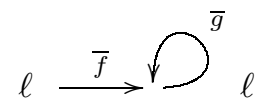

and hence $\ell \in$ fix $\bar{f} \cap$ fix $\bar{g}$. Thus $f^{*} \vee g^{*} \leq\left(f g^{*}\right)^{*}$, completing the proof of the first equality. The second is dual. 
We remark that the inequalities of the proposition are in general strict.

2.4.1. Finite lattices. Assume now that $L$ is a finite lattice. Then $\mathscr{C}\left(L^{2}\right)$ is a finite monoid. If $s$ is an element of a profinite semigroup, then $s^{\omega}$ denotes the unique idempotent in $\overline{\langle s\rangle}$. For $k \geq 0, s^{\omega+k}=s^{\omega} s^{k}$. For $k<0$, we denote by $s^{\omega-k}$ the inverse of $s^{\omega+k}$ in the procyclic group $\overline{\left\langle s^{\omega+1}\right\rangle}$.

Definition $2.38\left(()^{\omega+*}\right)$. If $L$ is a finite lattice, and $f \in \mathscr{C}\left(L^{2}\right)$, then we set

$$
f^{\omega+*}=f^{\omega} f^{*} \text {. }
$$

In other words, $\left(\ell, \ell^{\prime}\right)$ is stable for $f^{\omega+*}$ if and only if

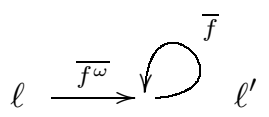

if and only if $\ell \stackrel{\overline{f^{\omega}}}{\longrightarrow} \ell^{\prime} \stackrel{\bar{f}}{\rightarrow} \ell^{\prime}$.

Remark 2.39. Notice that $f^{\omega} \leq f^{\omega+*}$ by Proposition 2.10 .

We establish a few basic properties of $f^{\omega+*}$.

Proposition 2.40. Let $g \in \mathscr{C}\left(L^{2}\right)$. Then $g^{\omega+*}$ and $g^{\omega+*} g^{\omega}$ are $\mathscr{R}$-equivalent idempotents. Moreover, $g^{\omega+*} g^{\omega} \leq g^{\omega+*}$.

Proof. Since $g^{\omega} \leq g^{*}$ by Proposition 2.37, evidently $g^{\omega+*} g^{\omega} \leq g^{\omega} g^{*} g^{*}=g^{\omega} g^{*}=$ $g^{\omega+*}$, establishing the second statement.

To prove the first statement we compute

$$
g^{\omega+*}=g^{\omega} g^{\omega+*} \leq g^{\omega+*} g^{\omega+*}=g^{\omega+*} g^{\omega} g^{*} \leq g^{\omega+*} g^{*}=g^{\omega+*} .
$$

Therefore, $g^{\omega+*} g^{\omega} g^{\omega+*}=g^{\omega+*} g^{\omega+*}=g^{\omega+*}$ and $g^{\omega+*} g^{\omega+*} g^{\omega}=g^{\omega+*} g^{\omega}$, whence $g^{\omega+*} \mathscr{R} g^{\omega+*} g^{\omega}$. Also, $g^{\omega+*} g^{\omega} g^{\omega+*} g^{\omega}=g^{\omega+*} g^{\omega}$.

The following lemma shows that $g^{\omega+*}$ and its dual absorb $g$.

Lemma 2.41. Let $L$ be a finite lattice and $g \in \mathscr{C}\left(L^{2}\right)$. Then $g g^{\omega+*}=g^{\omega+*}$ and dually $g^{*} g^{\omega} g=g^{*} g^{\omega}$. Hence

$$
g^{\omega+*} g^{\omega} g=g^{\omega+*} g^{\omega}=g g^{\omega+*} g^{\omega} .
$$

Proof. Equation (2.7) is an immediate consequence of the first part of the lemma. We just prove $g g^{\omega+*}=g^{\omega+*}$ as the other equality is dual. Indeed, Proposition 2.37 yields

$$
\begin{aligned}
g g^{\omega+*} & =g g^{\omega} g^{*}=g^{\omega} g g^{*} \\
& \leq g^{\omega} g^{*} g^{*}=g^{\omega+*}=g^{\omega+1} g^{\omega-1} g^{*} \\
& \leq g^{\omega+1} g^{*} g^{*}=g g^{\omega} g^{*}=g g^{\omega+*}
\end{aligned}
$$

as required.

When trying to establish the companion upper bound to the lower bound introduced in this paper, it will often be necessary to work with a "conjugated" version of $f^{\omega+*}$. 
Theorem 2.42 (Conjugated star). Let $f, g \in \mathscr{C}\left(L^{2}\right)$ and consider the L-automaton $\mathscr{A}$ :

$$
q_{0} \stackrel{(f g)^{\omega}}{\longrightarrow} q_{1} \underset{f}{\stackrel{g}{\longrightarrow}} q_{2} .
$$

Then $\mathscr{A}\left(q_{0}, q_{2}\right)=f(g f)^{\omega+*}$.

Proof. Suppose first that $\left(\ell, \ell^{\prime}\right)$ is stable for $\mathscr{A}\left(q_{0}, q_{2}\right)$. Then by Proposition 2.33 we can find $\ell^{\prime \prime} \in L$ so that

$$
\ell \stackrel{\overline{(f g)^{\omega}}}{\longrightarrow} \ell^{\prime \prime} \stackrel{\bar{g}}{\underset{\bar{f}}{\leftrightarrows}} \ell^{\prime}
$$

and hence we can find $\ell_{0}, \ell_{1} \in L$ such that

$$
\ell \stackrel{\bar{f}}{\rightarrow} \ell_{0} \stackrel{\bar{g}}{\rightarrow} \ell_{1} \stackrel{\overline{(f g)^{\omega-1}}}{\longrightarrow} \ell^{\prime \prime} \stackrel{\bar{f}}{\rightarrow} \ell^{\prime} \stackrel{\overline{g f}}{\longrightarrow} \ell^{\prime} .
$$

Composing, we obtain

$$
\left.\ell \stackrel{\bar{f}}{\longrightarrow} \ell_{0} \stackrel{\overline{(g f)^{\omega}}}{\longrightarrow} \ell^{\prime}\right)^{\overline{g f}}
$$

and so $\ell \overline{f(g f)^{\omega+*}} \ell^{\prime}$, as required.

Conversely, assume $\left(\ell, \ell^{\prime}\right)$ is stable for $f(g f)^{\omega+*}$. Lemma 2.41 yields $f(g f)^{\omega+*}=$ $f(g f)^{\omega-1}(g f)^{\omega+*}$ and so we can find $\ell_{1}, \ell_{2} \in L$ such that

$$
\ell \stackrel{\overline{f(g f)^{\omega-1}}}{\longrightarrow} \ell_{1} \stackrel{\overline{(g f)^{\omega}}}{\longrightarrow} \ell^{\prime} \stackrel{\bar{g}}{\rightarrow} \ell_{2} \stackrel{\bar{f}}{\rightarrow} \ell^{\prime} .
$$

Because $f(g f)^{\omega-1}(g f)^{\omega} g=(f g)^{\omega}$, we conclude

$$
\ell \stackrel{\overline{(f g)^{\omega}}}{\longrightarrow} \ell_{2} \underset{\bar{f}}{\stackrel{\bar{g}}{\longrightarrow}} \ell^{\prime}
$$

and so $\ell \stackrel{\overline{\mathscr{A}\left(q_{0}, q_{2}\right)}}{\longrightarrow} \ell^{\prime}$, again by Proposition 2.33, completing the proof.

\section{The Presentation Lemma: Flow form}

The main tool for dealing with complexity is the Presentation Lemma. We shall use the version of [30, Section 4.14] (see also 35]), rather than that of 6 . The key difference is that [6] views $R$ as $G \times B$, where $G$ is the maximal subgroup of $R$ and $B$ is the set of $\mathscr{H}$-classes of $R$ and uses the so-called Dowling lattice [7] (discovered independently by the second author) instead of the set-partition lattice. The goal of this section is to prove the following result, where $\mathrm{m}$ denotes the $\mathrm{Mal}^{\prime}$ cev product and $\operatorname{RLM}(M)$ is the right letter mapping image of $M$ (see below or 30]).

Theorem (Presentation Lemma: Flow form). Let $M$ be a finite $X$-generated group mapping monoid with distinguished $\mathscr{R}$-class $R$. Let $\mathbf{V}$ be a pseudovariety. Then $M \in \mathbf{A}(\mathbf{m})(\mathbf{G} * \mathbf{V})$ if and only if $\operatorname{RLM}(M) \in \mathbf{A}(\mathbf{m}(\mathbf{G} * \mathbf{V})$ and there exists a complete $\mathrm{SP}(M, X)$-flow $F$ on a partial automaton $\mathscr{A}$ over $X$ with transition monoid in $\mathbf{V}$ such that if $r, s$ are in the same block of $q F$ for some state $q$ and $r \mathscr{H} s$, then $r=s$. 
The aim of this section is to show that the statement of the above theorem is equivalent to the Presentation Lemma as stated in [30, Theorem 4.14.19]. The reader who is willing to accept this as a fact may skip ahead to Theorem 3.5

In this paper, we mean by a transformation semigroup a faithful partial transformation semigroup as per [9]. A relational morphism of partial transformation semigroups $\varphi:(R, M) \rightarrow(Q, N)$ is a fully defined relation $\varphi: R \rightarrow Q$ such that, for all $m \in M$, there exists $\widetilde{m} \in N$ so that

$$
q \varphi^{-1} m \subseteq(q \tilde{m}) \varphi^{-1}
$$

for all $q \in Q$. One says in this case that $\tilde{m}$ covers $m$. There is a companion relational morphism $\varphi^{c}: M \rightarrow N$ defined by

$$
m \varphi^{c}=\{n \in N \mid n \text { covers } m\} .
$$

A parameterized relational morphism of partial transformation semigroups $\Phi$ : $(R, M) \rightarrow(Q, N)$ is a pair $\left(\varphi_{0}, \varphi_{1}\right)$, where $\varphi_{0}:(R, M) \rightarrow(Q, N)$ and $\varphi_{1}: M \rightarrow N$ are relational morphisms such that $\varphi_{1} \subseteq \varphi_{0}^{c}$, that is, each $n \in m \varphi_{1}$ covers $m$. Suppose that $M$ and $N$ are both $X$-generated. Then the parameterized relational morphism is termed canonical if $\varphi_{1}$ is the relational morphism whose graph is generated by all pairs of the form $\left([x]_{M},[x]_{N}\right)$ with $x \in X$. Here we use the convention that if $M$ is an $X$-generated monoid and $w \in X^{*}$, then $[w]_{M}$ is the image of $w \in M$. Sometimes, we just write $w$ if $M$ is understood.

Let $\Phi:(R, M) \rightarrow(Q, N)$ be a parameterized relational morphism. We shall need the following partial automaton, denoted $\mathcal{D}_{\Phi}$, which is in fact the automaton underlying the derived transformation semigroup of $\Phi$ [9] (without the empty function) viewed as an automaton. The state set of $\mathcal{D}_{\Phi}$ is

$$
\# \varphi_{0}=\left\{(r, q) \mid q \in r \varphi_{0}\right\} .
$$

The transitions are of the form

$$
(r, q) \stackrel{(q,(m, n))}{\longrightarrow}(r m, q n),
$$

where $n \in m \varphi_{1}$ and $r m \in R$.

By an automaton congruence on a partial automaton $\mathscr{A}=(Q, X)$ we mean an equivalence relation $\equiv$ on $Q$ such that $q \equiv q^{\prime}$ and $q x, q^{\prime} x \in Q$ implies $q x \equiv q^{\prime} x$, for $q, q^{\prime} \in Q, x \in X$. The quotient automaton $\mathscr{A} / \equiv$ has state set $Q / \equiv$ and input alphabet $X$. There is a transition $[q] \stackrel{x}{\rightarrow}\left[q^{\prime}\right]$ if and only if there are $q_{0}, q_{0}^{\prime} \in Q$ with $q_{0} \in[q], q_{0}^{\prime} \in\left[q^{\prime}\right]$ and $q_{0} \stackrel{x}{\rightarrow} q_{0}^{\prime}$. The automaton congruence is called injective if $q x, q^{\prime} x \in Q$ and $q x \equiv q^{\prime} x$ implies $q \equiv q^{\prime}$, that is, if the transitions of the quotient automaton $\mathscr{A} / \equiv$ are partial one-to-one maps.

An automaton congruence, and its associated partition, on $\mathcal{D}_{\Phi}$ is called admissible if it is injective and

$$
(r, q) \equiv\left(r^{\prime}, q^{\prime}\right) \Longrightarrow q=q^{\prime}
$$

for $r, r^{\prime} \in R$ and $q, q^{\prime} \in Q$.

Suppose now that $M$ is a finite group mapping monoid with distinguished $\mathscr{R}$-class $R$. Let $B$ be the set of $\mathscr{L}$-classes of $M$. Then $M$ acts by partial transformations on $B$ via right multiplication, resulting in a transformation semigroup $(B, \operatorname{RLM}(M))$. Following [17, 30], RLM $(M)$ is called the right letter mapping semigroup of $M$. Since $R$ contains a non-trivial group, $\operatorname{RLM}(M)$ is always a proper image of $M[17,30,6]$. 
Definition 3.1 (Presentation). Let $\mathbf{V}$ be a pseudovariety of monoids. Then a presentation for $(R, M)$ over $\mathbf{V}$ is a pair $(\Phi, \mathscr{P})$, where $\Phi:(R, M) \rightarrow(Q, N)$ is a parameterized relational morphism, $N \in \mathbf{V}$ and $\mathscr{P}$ is an admissible partition on $\mathcal{D}_{\Phi}$ such that

$$
(r, q) \mathscr{P}(s, q) \text { and } r \mathscr{H} s \Longrightarrow r=s
$$

for $r, s \in R$ and $q \in Q$.

The following result is the Presentation Lemma [30, Theorem 4.14.19], originally due to the second author [6]. Recall that $\mathbf{A}$ denotes the pseudovariety of aperiodic monoids and $\mathbf{G}$ denotes the pseudovariety of finite groups. If $\mathbf{V}$ and $\mathbf{W}$ are pseudovarieties, $\mathbf{V} * \mathbf{W}$ denotes their semidirect product 9 , 30] and $\mathbf{V}(\mathrm{m}) \mathbf{W}$ their $\mathrm{Mal}^{\prime}$ cev product [11, 30].

Theorem 3.2 (Presentation Lemma). Let $M$ be a finite group mapping monoid and $\mathbf{V}$ be a pseudovariety. Then $M \in \mathbf{A}(\mathbf{m}(\mathbf{G} * \mathbf{V})$ if and only if $\operatorname{RLM}(M) \in \mathbf{A}(\mathbf{m}(\mathbf{G} * \mathbf{V})$ and $(R, M)$ has a presentation over $\mathbf{V}$ where $R$ is the distinguished $\mathscr{R}$-class of $M$.

Let $\mathbf{C}_{n}$ denote the pseudovariety of monoids of complexity at most $n$ [9, 30]. The Fundamental Lemma of Complexity [21, 40] shows that

$$
\mathbf{C}_{n}=\mathbf{A} \text { (m) }\left(\mathbf{G} * \mathbf{C}_{n-1}\right) \text {. }
$$

It is also a well-known consequence of the Fundamental Lemma of Complexity that the decidability of complexity reduces to the case of group mapping monoids; see the discussion in 6 . The above theorem, with $\mathbf{V}=\mathbf{C}_{n-1}$, shows that decidability of complexity $n$ reduces to the decidability of the existence of presentations over $\mathbf{C}_{n-1}$ for group mapping monoids. Here we are using the fact that we can assume by induction on order that membership of $\operatorname{RLM}(M)$ in $\mathbf{C}_{n}$ can already be determined.

There is also a stronger version of Theorem 3.2 Recall that if $M$ is a monoid and $\mathbf{V}$ is a pseudovariety, then a subset $A \subseteq M$ is called $\mathbf{V}$-pointlike if, for all relational morphisms $\varphi: M \rightarrow N$ with $N \in \mathbf{V}$, there exists $n \in N$ with $A \subseteq n \varphi^{-1}$ [30. The Presentation Lemma for pointlikes is due to the third author [35] and is [30, Theorem 4.14.20].

Theorem 3.3 ( $\mathbf{G} * \mathbf{V}$-pointlikes). Let $M$ be a finite group mapping monoid with distinguished $\mathscr{R}$-class $R$. Let $\mathbf{V}$ be a pseudovariety. Then $A \subseteq R$ is $\mathbf{G} * \mathbf{V}$-pointlike if and only if, for every parameterized relational morphism $\Phi:(R, M) \rightarrow(Q, N)$ with $N \in \mathbf{V}$ and every admissible partition $\mathscr{P}$ on $\mathcal{D}_{\Phi}$, there exists $q \in Q$ such that $A \subseteq q \varphi_{0}^{-1}$ and $A \times\{q\}$ is contained in a single block of $\mathscr{P}$.

We now wish to show how to go between set-partition flows and parameterized relational morphisms with admissible partitions on their derived automata.

Proposition 3.4. Let $M$ be an $X$-generated group mapping monoid with distinguished $\mathscr{R}$-class $R$.

(1) Suppose $\mathscr{A}=(Q, X)$ is a partial automaton with transition monoid $N$ and let $F: Q \rightarrow \mathrm{SP}(M, X)$ be a complete flow on $\mathscr{A}$. Then there exist a parameterized relational morphism $\Phi:(R, M) \rightarrow(Q, N)$ and an admissible partition $\mathscr{P}$ on $\mathcal{D}_{\Phi}$ so that if $q F=(A, P)$, then $q \varphi_{0}^{-1}=A$ and $(r, q) \mathscr{P}$ $\left(r^{\prime}, q\right)$, for $r, r^{\prime} \in A$, if and only if $r P r^{\prime}$.

(2) If $\Phi:(R, M) \rightarrow(Q, N)$ is a parameterized relational morphism and $\mathscr{P}$ is an admissible partition on $\mathcal{D}_{\Phi}$, then we can find a partial automaton $\mathscr{A}=(Q, X)$ with transition monoid a submonoid of $N$ and a complete flow 
$F$ on $\mathscr{A}$ so that, for all $q \in Q, q F=\left(q \varphi_{0}^{-1}, P_{q}\right)$, where $r P_{q} r^{\prime}$ if and only if $(r, q) \mathscr{P}\left(r^{\prime}, q\right)$.

Proof. We begin with (1). Define a canonical parameterized relational morphism $\Phi:(R, M) \rightarrow(Q, N)$ by putting $q \varphi_{0}^{-1}=A$, where $q F=(A, P)$. To see that $\varphi_{0}$ is fully defined, let $r \in R$. Since $F$ is a complete flow, there exists $q$ with $(r, r) \leq q F$. Then $r \in q \varphi_{0}^{-1}$. Next, we show that $[x]_{N}$ covers $[x]_{M}$. Indeed, suppose $q \in Q$ and $x \in X$. Let $q F=(A, P)$. Assume first that $q x=\emptyset$. Then since $F$ is a complete flow, we must have $A x=\emptyset$. Thus $q \varphi_{0}^{-1}[x]_{M}=A x=\emptyset \subseteq q[x]_{N} \varphi_{0}^{-1}$. Next suppose $q x \neq \emptyset$. Let $(q x) F=\left(A^{\prime}, P^{\prime}\right)$. Then, since $F$ is a flow, $A x \subseteq A^{\prime}$. Hence $q \varphi_{0}^{-1}[x]_{M}=A x \subseteq A^{\prime}=\left(q[x]_{N}\right) \varphi_{0}^{-1}$. Thus $[x]_{N}$ covers $[x]_{M}$, from which it follows by an easy induction that $[w]_{N}$ covers $[w]_{M}$ for all $w \in X^{*}$. So $\Phi$ is indeed a canonical parameterized relational morphism.

Next define a partition $\mathscr{P}$ on $\mathcal{D}_{\Phi}$ by setting $(r, q) \mathscr{P}\left(r^{\prime}, q\right)$ if $q F=(A, P)$ with $r, r^{\prime} \in A$ and $r P r^{\prime}$. Our goal is to verify that $\mathscr{P}$ is an admissible partition. It is immediate that $\mathscr{P}$ is a partition. To see it is an automaton congruence, we prove by induction on length that if $w \in X^{*}$ and $(r, q) \mathscr{P}\left(r^{\prime}, q\right)$ are such that $(r, q)\left(q,\left([w]_{M},[w]_{N}\right)\right)$ and $\left(r^{\prime}, q\right)\left(q,\left([w]_{M},[w]_{N}\right)\right)$ are defined, then $\left(r[w]_{M}, q[w]_{N}\right) \mathscr{P}\left(r^{\prime}[w]_{M}, q[w]_{N}\right)$. This is trivial if $|w|=0$. Suppose it is true for all words of length at most $n$ and suppose $w=u x$ with $|u|=n$ and $x \in X$. By induction, $\left(r[u]_{M}, q[u]_{N}\right) \mathscr{P}\left(r^{\prime}[u]_{M}, q[u]_{N}\right)$. Suppose $q u F=(A, P)$ and $q w F=q u x F=\left(A^{\prime}, P^{\prime}\right)$. Then $r[u]_{M} P r^{\prime}[u]_{M}$. Since $F$ is a flow, $A x \subseteq A^{\prime}$, whence $r[u]_{M} x, r^{\prime}[u]_{M} x \in A^{\prime}$ and $r[u]_{M} x \quad P^{\prime} r^{\prime}[u]_{M} x$. Thus $\left(r[w]_{M}, q[w]_{N}\right) \mathscr{P}$ $\left(r^{\prime}[w]_{M}, q[w]_{N}\right)$ and so $\mathscr{P}$ is an automaton congruence.

To see that $\mathscr{P}$ is injective, we establish by induction on length that if $w \in X^{*}$, $\left(q_{0},\left([w]_{M},[w]_{N}\right)\right)$ is defined on $(r, q),\left(r^{\prime}, q^{\prime}\right)$ and

$$
(r, q)\left(q_{0},\left([w]_{M},[w]_{N}\right)\right) \mathscr{P}\left(r^{\prime}, q^{\prime}\right)\left(q_{0},\left([w]_{M},[w]_{N}\right)\right),
$$

then $(r, q) \mathscr{P}\left(r^{\prime}, q^{\prime}\right)$. First note that we must have $q=q_{0}=q^{\prime}$. So if $|w|=0$, there is nothing to prove. Suppose the claim is true for all words of length at most $n$ and consider $w=u x$ with $x \in X$ and $|u|=n$. Set $p=q u$. Then we have

$$
\begin{aligned}
\left(r[u]_{M}, p\right)\left(p,\left([x]_{M},[x]_{N}\right)\right) & =(r, q)\left(q_{0},\left([w]_{M},[w]_{N}\right)\right) \\
& \mathscr{P}\left(r^{\prime}, q^{\prime}\right)\left(q_{0},\left([w]_{M},[w]_{N}\right)\right) \\
& =\left(r^{\prime}[u]_{M}, p\right)\left(p,\left([x]_{M},[x]_{N}\right)\right) .
\end{aligned}
$$

Let $(A, P)=p F$ and $\left(A^{\prime}, P^{\prime}\right)=p x F$. As $F$ is a complete flow, $A x \subseteq A^{\prime}$ and

$$
A / P \stackrel{\cdot x}{\longrightarrow} A^{\prime} / P^{\prime} \text { is a partial injective map. }
$$

Thus $r[u x]_{M}, r^{\prime}[u x]_{M} \in A^{\prime}$. Because $\left(r[w]_{M}, q[w]_{N}\right) \mathscr{P}\left(r^{\prime}[w]_{M}, q^{\prime}[w]_{N}\right)$, it follows from the definition that $r[u x]_{M}=r[w]_{M} P^{\prime} r^{\prime}[w]_{M}=r^{\prime}[u x]_{M}$. Therefore, $r[u]_{M} P$ $r^{\prime}[u]_{M}$ by (3.2) and hence $\left(r[u]_{M}, p\right) \mathscr{P}\left(r^{\prime}[u]_{M}, p\right)$. Induction now yields $(r, q) \mathscr{P}$ $\left(r^{\prime}, q^{\prime}\right)$, as required. So $\mathscr{P}$ is an injective automaton congruence. It is admissible directly from the definition, establishing (1).

For (2), suppose that $\Phi=\left(\varphi_{0}, \varphi_{1}\right)$. Fix, for each $x \in X$ an element $n_{x} \in[x]_{M} \varphi_{1}$. Define a partial deterministic automaton $\mathscr{A}$ with state set $Q$ and transitions given by $q \stackrel{x}{\rightarrow} q n_{x} ;$ the transition monoid of $\mathscr{A}$ is

$$
\left\langle n_{x} \mid x \in X\right\rangle \leq N .
$$


Define a flow $F: Q \rightarrow \operatorname{SP}(M, X)$ by setting $q F=\left(q \varphi_{0}^{-1}, P_{q}\right)$, where $r P_{q} r^{\prime}$ if and only if $(r, q) \mathscr{P}\left(r^{\prime}, q\right)$. To see that $F$ is fully defined, observe that if $r \in R$, then there exists $q \in Q$ so that $r \in q \varphi_{0}^{-1}$. Then $(r, r) \leq q F$. Suppose that $q x=\emptyset$ and let $(A, P)=q F$. Then

$$
A x=q \varphi_{0}^{-1} x \subseteq q n_{x} \varphi_{0}^{-1}=q x \varphi_{0}^{-1}=\emptyset
$$

and so $A x=\emptyset$, whence $q F \stackrel{\bar{x}}{\rightarrow} B$. Thus $F$ is complete. It remains to verify that $F$ is a flow. Suppose that $q \stackrel{x}{\rightarrow} q x$ is a transition. Then we have

$$
q \varphi_{0}^{-1} x \subseteq q n_{x} \varphi_{0}^{-1}=q x \varphi_{0}^{-1}
$$

since $n_{x} \in[x]_{M} \varphi_{1}^{-1}$ and $\Phi$ is a parameterized relational morphism.

To ease notation, set $A=q \varphi_{0}^{-1}$ and $A^{\prime}=q x \varphi_{0}^{-1}$. Then (3.3) implies that $A x \subseteq A^{\prime}$. Let $\pi: \# \varphi_{0} \rightarrow R$ and $\rho: \mathcal{D}_{\Phi} \rightarrow \mathcal{D}_{\Phi} / \mathscr{P}$ be the projections. There then results the commutative diagram in Figure 1, where the vertical lines are bijections

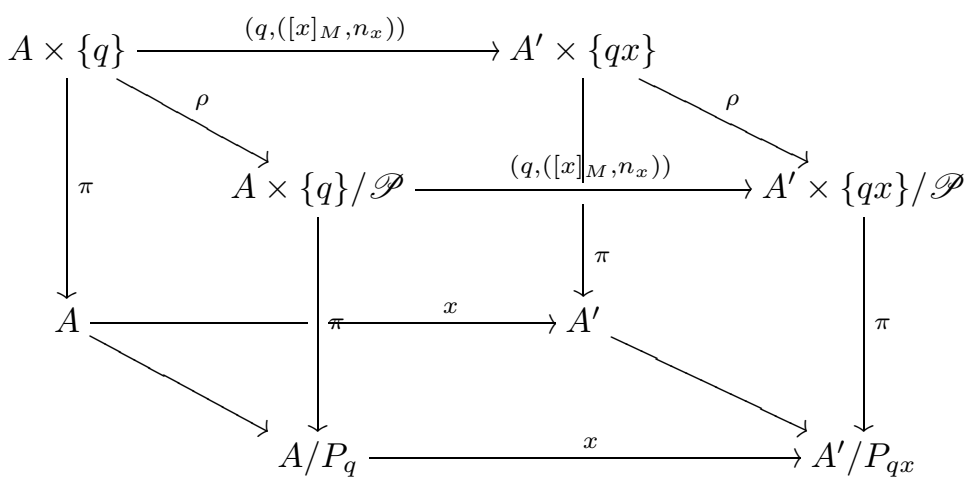

Figure 1. A commutative diagram showing $F$ is a flow

and the unlabelled arrows are the projections. Since $\mathscr{P}$ is an injective automaton congruence, it now follows that $A / P_{q} \stackrel{\cdot x}{\rightarrow} A^{\prime} / P_{q x}$ is a partial injective map. This completes the proof that $F$ is a flow, thereby establishing (2).

The above proposition easily leads to the following two theorems, which are restatements of Theorems 3.2 and 3.3 in the language of flows.

Theorem 3.5 (Presentation Lemma: Flow form). Let $M$ be a finite $X$-generated group mapping monoid with distinguished $\mathscr{R}$-class $R$. Let $\mathbf{V}$ be a pseudovariety. Then $M \in \mathbf{A} \mathrm{M}(\mathbf{G} * \mathbf{V})$ if and only if

$$
\operatorname{RLM}(M) \in \mathbf{A}(\mathbf{m})(\mathbf{G} * \mathbf{V})
$$

and there exists a complete $\mathrm{SP}(M, X)$-flow $F$ on a partial automaton $\mathscr{A}$ over $X$ with transition monoid in $\mathbf{V}$ such that if $(\{r, s\},\{\{r, s\}\}) \leq q F$ for some state $q$ (i.e., $r, s$ belong to the same block of $q f$ ) and $r \mathscr{H} s$, then $r=s$.

Proof. Suppose first that such a flow $F$ exists. Then $(\Phi, \mathscr{P})$ constructed in Proposition 3.4 (1) is a presentation over V. Conversely, if $(\Phi, \mathscr{P})$ is a presentation over $\mathbf{V}$, then Proposition 3.4(2) defines the desired flow. The result now follows from Theorem 3.2. 
The next theorem can be deduced from Theorem 3.3 in an analogous fashion; we omit the proof.

Theorem 3.6 ( $\mathbf{G} * \mathbf{V}$-pointlikes: Flow form). Let $M$ be a finite $X$-generated group mapping monoid with distinguished $\mathscr{R}$-class $R$. Let $\mathbf{V}$ be a pseudovariety. Then $A \subseteq R$ is $\mathbf{G} * \mathbf{V}$-pointlike if and only if, for every complete $\mathrm{SP}(M, X)$-flow on a partial automaton $\mathscr{A}$ over $X$ with transition monoid in $\mathbf{V}$, there is a state $q$ such that $(A,\{A\}) \leq q f$, i.e., $A$ is contained in a single block of $q f$.

So computing membership in $\mathbf{C}_{n}$ amounts to studying set-partition flows on automata with transition monoid in $\mathbf{C}_{n-1}$.

3.1. Inevitable set-partitions. In this section, we define the notion of an inevitable set-partition with respect to a pseudovariety $\mathbf{V}$. Throughout this section, we put $L=\mathrm{SP}(M, X)$.

Definition 3.7 ( $\mathbf{V}$-inevitability). Let $\mathbf{V}$ be a pseudovariety. Then an element $\ell \in L$ is said to be $\mathbf{V}$-inevitable if, for all complete flows $F$ on a partial automaton $\mathscr{A}=(Q, X)$ with transition monoid in $\mathbf{V}$, there is a state $q \in Q$ such that $\ell \leq q F$.

Notice that the set of $\mathbf{V}$-inevitable elements of $L$ is an order ideal. That is, if $\ell^{\prime} \leq \ell$ and $\ell$ is $\mathbf{V}$-inevitable, then so is $\ell^{\prime}$. Also notice that the points of $L$ are $\mathrm{V}$-inevitable by definition of a complete flow. The importance of this notion comes from the following reformulation of Theorem 3.6 .

Corollary 3.8. Let $M$ be a finite $X$-generated group mapping monoid with distinguished $\mathscr{R}$-class $R$. Let $\mathbf{V}$ be a pseudovariety. Then $A \subseteq R$ is $\mathbf{G} * \mathbf{V}$-pointlike if and only if there is a $\mathbf{V}$-inevitable element $(Y, P) \in \operatorname{SP}(L)$ such that $A \subseteq Y$ and $A$ is contained in a block of $P$, i.e., $(A,\{A\}) \leq(Y, P)$.

Proof. Theorem 3.6 says exactly that $A$ is $\mathbf{G} * \mathbf{V}$-pointlike if and only if $(A,\{A\})$ is $\mathbf{V}$-inevitable. Since the set of $\mathbf{V}$-inevitable set-partitions is an order ideal, this completes the proof.

The following standard compactness result is called the "Little Boxes Theorem" by the second author (the boxes refer to the blocks of the partition in a set-partition flow).

Theorem 3.9 (Little Boxes Theorem). Let $\mathbf{V}$ be a pseudovariety. Then there is a partial automaton $\mathscr{A}=(Q, X)$ with transition monoid in $\mathbf{V}$ and a complete flow $F$ on $\mathscr{A}$ such that $\ell \in L$ is $\mathbf{V}$-inevitable if and only if $\ell \leq q F$ for some $q \in Q$.

Proof. Since $L$ is finite, the set $W$ of elements of $L$ that are not $\mathbf{V}$-inevitable is finite. For each $\ell \in W$, we can find a partial automaton $\mathscr{A}_{\ell}=\left(Q_{\ell}, X\right)$ with transition monoid in $\mathbf{V}$ and a complete flow $F_{\ell}$ on $\mathscr{A}_{\ell}$ such that $\ell \not \leq q F_{\ell}$ for all $q \in Q$. Let $\mathscr{A}=\prod_{\ell \in W} \mathscr{A}_{\ell}=(Q, X)$. Then $\mathscr{A}$ has transition monoid in $\mathbf{V}$. Define $F: Q \rightarrow L$ by

$$
\left(q_{\ell}\right) F=\bigwedge_{\ell \in W} q_{\ell} F_{\ell}
$$

Suppose that $\left(q_{\ell}\right) \stackrel{x}{\rightarrow}\left(q_{\ell}^{\prime}\right)$ is an edge of $\mathscr{A}$. Then, for each $\ell \in L, q_{\ell} \stackrel{x}{\rightarrow} q_{\ell}^{\prime}$. So $q_{\ell} F_{\ell} \stackrel{\bar{x}}{\rightarrow} q_{\ell}^{\prime} F_{\ell}$ for all $\ell \in W$. Since $\stackrel{\bar{x}}{\rightarrow}$ is meet-closed, it follows that $\left(q_{\ell}\right) F \stackrel{\bar{x}}{\rightarrow}\left(q_{\ell}^{\prime}\right) F$ and hence $F$ is a flow. 
We must now check that $F$ is a complete flow. To see that $F$ is fully defined, let $r \in R$. Then, for each $\ell \in W$, there is a state $q_{\ell}$ with $(r, r) \leq q_{\ell} F_{\ell}$ since the $F_{\ell}$ are complete flows. Hence

$$
(r, r) \leq \bigwedge_{\ell \in W} q_{\ell} F_{\ell}=\left(q_{\ell}\right) F
$$

establishing that $F$ is fully defined.

To see that $F$ extends to $\mathscr{A}^{\square}$, we must show that if $\left(q_{\ell}\right) x$ is not defined, then $\left(q_{\ell}\right) F \stackrel{\bar{x}}{\rightarrow} B$. But if $\left(q_{\ell}\right) x$ is not defined, then $q_{\ell^{\prime}} x$ is undefined for some $\ell^{\prime} \in W$. Hence

$$
q_{\ell^{\prime}} F_{\ell^{\prime}} \stackrel{\bar{x}}{\rightarrow} B
$$

since $F_{\ell^{\prime}}$ is a complete flow. Suppose $q_{\ell^{\prime}} F_{\ell^{\prime}}=\left(Y^{\prime}, P^{\prime}\right)$ and $\left(q_{\ell}\right) F=(Y, P)$. From the definitions, we have $(Y, P) \leq\left(Y^{\prime}, P^{\prime}\right)$. But $Y^{\prime} x=\emptyset$ by (3.5) and so $Y x=\emptyset$. Therefore, $\left(q_{\ell}\right) F \stackrel{\bar{x}}{\rightarrow} B$, establishing that $F$ is a complete flow.

To see that $F$ has the desired property, we must show that if $\ell \in W$, then $\ell \not \leq q F$ for all $q \in Q$. So suppose $\ell \in W$ and that, by way of a contradiction, $\ell \leq\left(q_{\ell^{\prime}}\right) F$. Then we have $\ell \leq q_{\ell^{\prime}} F_{\ell^{\prime}}$ for all $\ell^{\prime} \in W$ by (3.4). In particular, $\ell \leq q_{\ell} F_{\ell}$, a contradiction to the choice of $F_{\ell}$. It follows that $F$ has the desired property, establishing the theorem.

Combining Theorems 3.5 and 3.9 , one easily deduces the following corollary.

Corollary 3.10. Let $M$ be a finite $X$-generated group mapping monoid with distinguished $\mathscr{R}$-class $R$ and let $\mathbf{V}$ be a pseudovariety of monoids. Then $M \in \mathbf{A}(\mathbb{m}(\mathbf{G} * \mathbf{V})$ if and only if $\operatorname{RLM}(M) \in \mathbf{A}(\mathrm{m})(\mathbf{G} * \mathbf{V})$ and there are no $\mathbf{V}$-inevitable elements of the form $(\{r, s\},\{\{r, s\}\})$ with $r \mathscr{H} s$ and $r \neq s \in R$.

Proof. Suppose first that there are no $\mathbf{V}$-inevitable elements of the form $(\{r, s\}$, $\{\{r, s\}\})$ with $r \mathscr{H} s$ and $r \neq s \in R$. Then the flow provided in the Little Boxes Theorem satisfies the conditions of Theorem 3.5. Conversely, if there is a Vinevitable element of the form $(\{r, s\},\{\{r, s\}\})$ with $r \mathscr{H} s$ and $r \neq s$, then no flow satisfying the conditions of Theorem 3.5 can exist and therefore $M \notin \mathbf{A}(\mathrm{m})(\mathbf{G} * \mathbf{V})$ by Theorem 3.5 .

\section{THE FLOW MONOID}

In this section, we provide the tools for constructing an effective lower bound for complexity. The lower bound will be established in the next section. Again fix the notation $L=\operatorname{SP}(M, X)$. We write $I$ for $I_{L}$.

4.1. Loopable elements. To describe our lower bound, we need the notion of an $n$-loopable element of a monoid, which is defined inductively. First we need some definitions from [33; complete details can be found in [30, Section 4.12]. Denote by $\mathbf{R}$ the pseudovariety of $\mathscr{R}$-trivial semigroups and by $\mathbf{E R}$ the pseudovariety of semigroups whose idempotents generate an $\mathscr{R}$-trivial semigroup. Stiffler proved that $\mathbf{E R}=\mathbf{R} * \mathbf{G}$ [36, 1, 9]. In addition, we shall also require Stiffler's switching rule: $\mathbf{G} * \mathbf{R} \subseteq \mathbf{R} * \mathbf{G}$; see [30, Corollary 4.5.3].

Definition 4.1 (Type I). A submonoid $S^{\prime}$ of a monoid $S$ is said to be Type $I$ if, for any relational morphism $\varphi: S \rightarrow T$ with $T \in \mathbf{A}$, there exists a submonoid $T^{\prime} \leq T$ so that $T^{\prime} \in \mathbf{E R}$ and $S^{\prime} \subseteq T^{\prime} \varphi^{-1}$. 
A monoid is said to be absolute Type I if it is a Type I submonoid of itself. Absolute Type I monoids were effectively characterized by the first two authors, Margolis and Pin in [11; see [30, Theorem 4.12.19]. It follows from a result of the authors 13 that it is decidable whether a submonoid of a monoid is Type I. We briefly explain.

Definition 4.2 ( $\mathbf{V}$-stable pair). Let $S$ be a monoid and suppose that $A \subseteq S$ and $S^{\prime}$ is a submonoid of $S$. Then $\left(A, S^{\prime}\right)$ is called a $\mathbf{V}$-stable pair if, for all relational morphisms $\varphi: S \rightarrow T$ with $T \in \mathbf{V}$, there is an element $t \in T$ so that $A \subseteq t \varphi^{-1}$ and $S^{\prime} \subseteq \operatorname{Stab}(t) \varphi^{-1}$, where $\operatorname{Stab}(t)=\{u \in T \mid t u=t\}$ is the right stabilizer of $t$ in $T$.

The reader should not confuse stable pairs for closure operators on $L \times L$ with $\mathbf{V}$-stable pairs with respect to a pseudovariety $\mathbf{V}$. The latter will always be prefixed by the name of the pseudovariety. The following theorem was proved by the authors in [13].

Theorem 4.3. The set of A-stable pairs of a finite monoid is effectively constructible.

The next proposition relates $\mathbf{A}$-stable pairs to Type I submonoids. We shall make use of relatively free profinite monoids in the proof. Let $\mathbf{V}$ be a pseudovariety of monoids. If $X$ is a finite set, then $\widehat{F}_{\mathbf{V}}(X)$ denotes the free pro- $\mathbf{V}$ monoid on $X$ for $\mathbf{V}$ a pseudovariety of monoids [30, Chapter 3]. We write $F_{\mathbf{V}}^{\omega}(X)$ for the unary submonoid of $\widehat{F}_{\mathbf{V}}(X)$ generated by $X$ with ()$^{\omega}$ as the unary operation.

Proposition 4.4. Let $S$ be a finite monoid and $S^{\prime}$ a submonoid. Then the following are equivalent:

(1) $S^{\prime}$ is a Type I submonoid of $S$;

(2) there exists $s \in S$ so that $\left(\{s\}, S^{\prime}\right)$ is an $\mathbf{A}$-stable pair;

(3) there exists $\emptyset \neq A \subseteq S$ so that $\left(A, S^{\prime}\right)$ is an $\mathbf{A}$-stable pair.

Consequently, it is decidable whether a submonoid of $S$ is Type $I$.

Proof. Fix a generating set $X$ for $S$. Let $\rho_{\mathbf{A}}: S \rightarrow \widehat{F}_{\mathbf{A}}(X)$ be the canonical relational morphism: the graph of $\rho_{\mathbf{A}}$ is the closed submonoid of $S \times \widehat{F}_{\mathbf{A}}(X)$ generated by all pairs $\left([x]_{S}, x\right)$ with $x \in X$.

To see that (1) implies (2), we use [30, Corollary 3.7.5] to assert that there is a closed submonoid $T \leq \widehat{F}_{\mathbf{A}}(X)$ that is pro-ER so that $S^{\prime} \subseteq T \rho_{\mathbf{A}}^{-1}$. Let $J$ be the minimal ideal of $T$; then $J$ is pro-A (and so has trivial maximal subgroups) and has a unique $\mathscr{L}$-class. It follows from stability of profinite semigroups 30 , Proposition 3.1.10] that if $t \in J$ is any element, then $T \subseteq \operatorname{Stab}(t)$. So if $s \in t \rho_{\mathbf{V}}^{-1}$, then $\left(\{s\}, S^{\prime}\right)$ is an A-stable pair by [13, Theorem 2.6]. Clearly (2) implies (3). For (3) implies (1), we note that [13, Theorem 2.6] implies that there exists $t \in \widehat{F}_{\mathbf{A}}(X)$ so that $A \subseteq t \rho_{\mathbf{A}}^{-1}$ and $S^{\prime} \subseteq \operatorname{Stab}(t) \rho_{\mathbf{A}}^{-1}$. But [13, Theorem 4.1] shows that $\operatorname{Stab}(t)$ is a chain in its own (internal) $\mathscr{L}$-order and hence it must be $\mathscr{R}$-trivial since it is pro-A. Thus $S^{\prime}$ is a Type I submonoid of $S$ by another application of [30, Corollary 3.7.5].

The decidability result is immediate from the decidability of (2) or (3).

We also need the notion of Type II elements.

Definition 4.5 (Type II). An element $s$ of a monoid $S$ is said to be of Type II if, for all relational morphisms $\varphi: S \rightarrow G$ with $G \in \mathbf{G}$, one has $s \in 1 \varphi^{-1}$. Denote by $\mathrm{K}_{\mathrm{G}}(S)$ the set of all Type II elements of $S$; it is a submonoid. 
If $a, b \in S$ are such that $a b a=a$ and $s \in S$, then we say that $a s b, b s a$ are weak conjugates of $s$. The following effective characterization of Type II elements was conjectured by the second author and proved by Ash [4, and independently by Ribes and Zalesskii [34]; see [30, Theorem 4.17.30] or [5] for perhaps the easiest proofs.

Theorem 4.6. Let $S$ be a monoid. Then $\mathrm{K}_{\mathbf{G}}(S)$ is the least submonoid of $S$ closed under weak conjugation.

We now wish to define the notion of a $\mathbf{V}$-aperiodic element of a monoid, where $\mathrm{V}$ is a pseudovariety of monoids.

Definition 4.7 ( $\mathrm{V}$-aperiodic element). An element $s \in S$ of a profinite monoid is called aperiodic if $s^{\omega}=s^{\omega+1}$. If $\mathbf{V}$ is a pseudovariety of monoids, then an element $s$ of a monoid $S$ is called $\mathbf{V}$-aperiodic if, for all relational morphisms $\varphi: S \rightarrow T$ with $T \in \mathbf{V}$, there exists an aperiodic element $t \in T$ so that $t \in s \varphi$.

Remark 4.8. Notice that any element is A-aperiodic. An element of a monoid is G-aperiodic if and only if it is of Type II. It is obvious that if $S^{\prime} \leq S$ and $s$ is $\mathbf{V}$-aperiodic in $S^{\prime}$, then $s$ is $\mathbf{V}$-aperiodic in $S$. Also note that if $S \in \mathbf{V}$, then each $\mathbf{V}$-aperiodic element of $S$ must be, in fact, aperiodic (consider the identity homomorphism).

Recall from [30, Definition 3.6.25] that a subset $A$ of a finite monoid $S$ is said to be $\mathbf{V}$-like with respect to a pseudovariety $\mathbf{W}$ if, for all relational morphisms $\varphi: S \rightarrow W$ with $W \in \mathbf{W}$, there exists a submonoid $V \leq W$ so that $V \in \mathbf{V}$ and $A \subseteq V \varphi^{-1}$. It follows immediately from the definitions that a submonoid $S^{\prime}$ of $S$ is Type I if and only if it is ER-like with respect to $\mathbf{A}$ and that an element $s \in S$ is $\mathbf{V}$-aperiodic if and only if $\{s\}$ is $\mathbf{A}$-like with respect to $\mathbf{V}$. The following is then a special case of what is proved in [30, page 179].

Proposition 4.9. Let $S$ be a finite monoid and $\mathbf{V}$ a pseudovariety. Then there exists a relational morphism $\varphi: S \rightarrow V$ with $V \in \mathbf{V}$ such that $s \in S$ is $\mathbf{V}$-aperiodic if and only if there exists an aperiodic element $v \in V$ so that $v \in s \varphi$.

We aim to provide a computable set of $\mathbf{C}_{n}$-aperiodic elements. We begin with a straightforward reduction to a generating set for the pseudovariety. Recall that an injective relational morphism is called a division [9, 30.

Lemma 4.10. Let $\varphi: S \rightarrow T$ be a relational morphism and $d: T \rightarrow U$ a division. Fix $s \in S$ and suppose there is an aperiodic element $u \in U$ with $u \in s \varphi d$. Then there is an aperiodic element $t \in T$ so that $t \in s \varphi$.

Proof. Choose $t \in T$ so that $t \in s \varphi$ and $u \in t d$. We claim $t$ is aperiodic. Indeed, $u^{\omega} \in t^{\omega} d \cap t^{\omega+1} d$ and so $t^{\omega}=t^{\omega+1}$ as $d$ is a division.

The following lemma shows how to generate $\mathbf{V} * \mathbf{G} * \mathbf{A}$-aperiodic elements.

Lemma 4.11. Let $\mathbf{V}$ be a pseudovariety such that $\mathbf{V} * \mathbf{R}=\mathbf{V}$. Let $S^{\prime}$ be a Type I submonoid of a monoid $S$ and suppose that $s$ is a $\mathbf{V}$-aperiodic element of $\mathrm{K}_{\mathbf{G}}\left(S^{\prime}\right)$. Then $s$ is $\mathbf{V} * \mathbf{G} * \mathbf{A}$-aperiodic in $S$.

Proof. By Lemma 4.10 it suffices to show that if $\varphi: S \rightarrow B \rtimes A$ is a relational morphism with $B \in \mathbf{V} * \mathbf{G}$ and $A \in \mathbf{A}$, then there exists an aperiodic element $t \in B \rtimes A$ so that $s \in t \varphi^{-1}$. 
Let $\pi: B \rtimes A \rightarrow A$ be the semidirect product projection. Setting $\psi=\varphi \pi$, we can find a submonoid $A^{\prime} \leq A$ with $A^{\prime} \in \mathbf{E R}$ and $S^{\prime} \subseteq A^{\prime} \psi^{-1}$. Hence, by restricting $\varphi$, we can obtain a relational morphism $\eta: S^{\prime} \rightarrow B \rtimes A^{\prime}$ with $\eta \subseteq \varphi$. Set $T=B \rtimes A^{\prime}$. Then $T \in \mathbf{V} * \mathbf{G} * \mathbf{E R}$ and

$$
\mathbf{V} * \mathbf{G} * \mathbf{E R}=\mathbf{V} * \mathbf{G} * \mathbf{R} * \mathbf{G} \subseteq \mathbf{V} * \mathbf{R} * \mathbf{G} * \mathbf{G} \subseteq \mathbf{V} * \mathbf{G}
$$

and so $\mathbf{K}_{\mathbf{G}}(T) \in \mathbf{V}$. Choose a finite monoid $R$ so that $\eta=\alpha^{-1} \beta$ with $\alpha: R \rightarrow S^{\prime}$ an onto homomorphism and $\beta: R \rightarrow T$ a homomorphism. By [30, Proposition 4.12.6], we have that $\mathrm{K}_{\mathbf{G}}(R) \alpha=\mathrm{K}_{\mathbf{G}}\left(S^{\prime}\right)$ and $\mathrm{K}_{\mathbf{G}}(R) \beta \subseteq \mathrm{K}_{\mathbf{G}}(T)$. Hence, we obtain a relational morphism $\tau: \mathrm{K}_{\mathbf{G}}\left(S^{\prime}\right) \rightarrow \mathrm{K}_{\mathbf{G}}(T)$ with $\tau \subseteq \eta$. Since $\mathbf{K}_{\mathbf{G}}(T) \in \mathbf{V}$ and $s$ is $\mathrm{V}$-aperiodic, there exists an aperiodic element $t \in \overline{\mathrm{K}}_{\mathrm{G}}(T) \subseteq T$ so that $s \in t \tau^{-1} \subseteq$ $t \eta^{-1} \subseteq t \varphi^{-1}$. This completes the proof that $s$ is $\mathbf{V} * \mathbf{G} * \mathbf{A}$-aperiodic in $S$.

With Lemma 4.11 in hand, we can define recursively a class of $\mathbf{C}_{n}$-aperiodic elements, which we call $n$-loopable elements.

Definition 4.12 ( $n$-loopable element). An $n$-loopable element of a monoid $S$ is defined recursively as follows:

- Any element of $S$ is 0-loopable.

- An element $s \in S$ is $n$-loopable in $S$, for $n \geq 1$, if there exists a Type I submonoid $T$ of $S$ so that $s$ is an $(n-1)$-loopable element of $\mathrm{K}_{\mathbf{G}}(T)$.

Since one can effectively find all the Type I submonoids of a monoid and the Type II submonoid is effectively computable, it follows that the set of $n$-loopable elements of a monoid is effectively computable. An easy induction establishes that $n$-loopable elements are $\mathbf{C}_{n}$-aperiodic.

Proposition 4.13. Suppose that $s \in S$ is n-loopable. Then $s$ is $\mathbf{C}_{n}$-aperiodic.

Proof. We proceed by induction on $n$, the case $n=0$ being trivial since all elements are $\mathbf{A}$-aperiodic. Suppose the result is true for $n \geq 0$ and suppose $s \in S$ is $(n+1)$ loopable. Then we can find a Type I submonoid $T$ of $S$ so that $s$ is $n$-loopable in $\mathrm{K}_{\mathbf{G}}(T)$. By induction, $s$ is $\mathbf{C}_{n}$-aperiodic in $\mathrm{K}_{\mathbf{G}}(T)$ and so is $\mathbf{C}_{n} * \mathbf{G} * \mathbf{A}$-aperiodic in $T$ by Lemma 4.11. Thus $s$ is $\mathbf{C}_{n+1}$-aperiodic in $S$ by Remark 4.8 ,

Let us show that computing $\mathbf{C}_{n}$-aperiodic elements is tantamount to computing complexity.

Proposition 4.14. Let $\mathbf{V}$ be a pseudovariety of monoids. Then $s \in S$ is $\mathbf{V}$ aperiodic if and only if it is $\mathbf{A}(\mathrm{m}) \mathbf{V}$-aperiodic.

Proof. Clearly any $\mathbf{A}(\mathrm{m}) \mathbf{V}$-aperiodic element is $\mathbf{V}$-aperiodic. For the converse, by Lemma4.10 it suffices to consider relational morphisms $\varphi: S \rightarrow T$ so that $T$ admits an aperiodic homomorphism $\psi: T \rightarrow V$ with $V \in \mathbf{V}$. Let $v \in V$ be aperiodic with $v \in s \varphi \psi$. By Lemma [30, Lemma 4.4.4], $\langle v\rangle \psi^{-1}$ is aperiodic. Hence if $t \in T$ is such that $t \in s \varphi$ and $v=t \psi$, then $t$ is aperiodic.

Consequently, we have the following membership criterion for $\mathbf{A}(m) \mathbf{V}$.

Corollary 4.15. Let $\mathbf{V}$ be a pseudovariety. Then the following are equivalent:

(1) $S \in \mathbf{A}$ (m) $\mathbf{V}$.

(2) Each $\mathbf{A}(\mathbb{M} \mathbf{V}$-aperiodic element of $S$ is aperiodic.

(3) Each $\mathbf{V}$-aperiodic element of $S$ is aperiodic. 
Proof. The implication from (1) to (2) was mentioned in Remark 4.8 Trivially, (2) implies (3). Suppose (3) holds. Find a relational morphism $\varphi: S \rightarrow V$ with $V \in \mathbf{V}$ as per Proposition 4.9. Let $e \in V$ be an idempotent. Then since $e$ is aperiodic, each element of $e \varphi^{-1}$ is $\mathbf{V}$-aperiodic and hence aperiodic by assumption. Thus $S \in \mathbf{A}$ (m) V.

The Fundamental Lemma of Complexity [21, 40, 30] asserts that $\mathbf{C}_{n}=\mathbf{A}(\mathrm{m})(\mathbf{G} *$ $\mathbf{C}_{n-1}$ ), so we obtain the following consequence.

Corollary 4.16. A monoid $S$ belongs to $\mathbf{C}_{n}$ if and only if all its $\mathbf{C}_{n}$-aperiodic elements are aperiodic. Hence the complexity of $S$ is the least $n$ so that all $\mathbf{C}_{n}$ aperiodic elements of $S$ are aperiodic.

The next corollary is a rephrasing of the Type I-Type II lower bound of [33] in the language of loopable elements.

Corollary 4.17. Let $n$ be the least non-negative integer so that each $n$-loopable element of $S$ is aperiodic. Then $S$ has complexity at least $n$.

The Tall Fork from [30, Section 4.14] has the property that all its 1-loopable elements are aperiodic, but it has complexity 2 . Thus the $n$-loopable elements form a proper subset of the $\mathbf{C}_{n}$-aperiodic elements.

4.2. Values and inevitable flows. Fix again an $X$-generated group mapping monoid $M$ with distinguished $\mathscr{R}$-class $R$ and put $L=\mathrm{SP}(M, X)$. In this subsection we single out a certain submonoid of the abstract flow monoid consisting of so-called V-inevitable elements.

If $(Q, X)$ is a partial automaton with transition monoid in $\mathbf{V}$, then $\widehat{F}_{\mathbf{V}}(X)$ acts naturally on $Q$. In what follows, if $F$ is a complete flow on a partial automaton $\mathscr{A}$ and $q t$ is undefined for $q \in Q$, then we interpret $q t F$ as $\square F=B$.

Definition 4.18 ( $\mathrm{V}$-inevitable flow). Let $\mathrm{V}$ be a pseudovariety of monoids. An abstract flow $f \in \mathscr{C}\left(L^{2}\right)$ is said to be $\mathbf{V}$-inevitable if, for all complete flows $F$ on a partial automaton $\mathscr{A}=(Q, X)$ with transition monoid $N \in \mathbf{V}$, there exists $t \in N$ so that one has

$$
q F \stackrel{\bar{f}}{\rightarrow} q t F
$$

for all states $q \in Q$.

It turns out that, for any complete flow on an automaton with transition monoid in $\mathbf{V}$, the values of the flow are stable under back-flow by any $\mathbf{V}$-inevitable abstract flow.

Lemma 4.19. Let $F$ be a complete flow on a partial automaton $\mathscr{A}=(Q, A)$ with transition monoid $N \in \mathbf{V}$. Suppose that $f$ is a $\mathbf{V}$-inevitable abstract flow and $q \in Q$. Then $(q F) \overleftarrow{f}=q F$

Proof. Choose $t \in N$ so that (4.1) holds. Then evidently $q F \in \operatorname{dom} \bar{f}$ and hence is stable for $\overleftarrow{f}$

Proposition 4.20. Let $f$ be a $\mathbf{V}$-inevitable abstract flow. Then $B \stackrel{\bar{f}}{\rightarrow} B$. 
Proof. Consider the one-state complete automaton over $X$

$$
\mathscr{A}=q_{0} \bigcap^{X}
$$

and define a complete flow $F$ by $q_{0} F=B$. Then by the definition of $\mathbf{V}$-inevitability $B=q_{0} F \stackrel{\bar{f}}{\rightarrow} q_{0} F=B$ since the transition monoid is trivial.

It turns out that one can switch the order of the quantifiers in Definition 4.18,

Proposition 4.21. An abstract flow $f \in \mathscr{C}\left(L^{2}\right)$ is $\mathbf{V}$-inevitable if and only if, for all partial automata $\mathscr{A}=(Q, X)$ with transition monoid $N \in \mathbf{V}$, there exists $t \in N$ so that, for all complete flows $F$ on $\mathscr{A}$, one has

$$
q F \stackrel{\bar{f}}{\rightarrow} q t F
$$

for all states $q \in Q$.

Proof. Trivially, if the condition given in the proposition is verified, then $f$ is $\mathbf{V}$ inevitable. For the converse, assume $f$ is $\mathbf{V}$-inevitable and suppose $\mathscr{A}=(Q, X)$ is a partial automaton with transition monoid $N \in \mathbf{V}$. Let $\left\{F_{1}, \ldots, F_{m}\right\}$ be the finite set of all complete flows on $\mathscr{A}$. Consider the partial automaton $\mathscr{A}^{\prime}=(Q \times$ $\{1, \ldots, m\}, X)$ whose transitions, for $x \in X$, are of the form $(q, i) x=(q x, i)$ if $q x$ is defined and undefined otherwise. In other words, $\mathscr{A}^{\prime}$ is a disjoint union of $m$ copies of $\mathscr{A}$. Evidently, the transition monoid of $\mathscr{A}^{\prime}$ is $N$. Define a flow $F$ on $\mathscr{A}^{\prime}$ by $(q, i) F=q F_{i}$. It is straightforward to verify that $F$ is a complete flow. Since $f$ is $\mathbf{V}$-inevitable, there exists $t \in N$ so that

$$
q F_{i}=(q, i) F \stackrel{\bar{f}}{\rightarrow}(q, i) t F=q t F_{i}
$$

for all $q \in Q$ and $i=1, \ldots, m$. This completes the proof.

As with many inevitability notions, there is always a finite model witnessing $\mathrm{V}$-inevitable flows.

Proposition 4.22. There exists a complete flow $F$ on a finite partial automaton $\mathscr{A}$ with transition monoid $N \in \mathbf{V}$ so that $f \in \mathscr{C}\left(L^{2}\right)$ is $\mathbf{V}$-inevitable if and only if there exists $t \in T$ so that (4.1) holds.

Proof. Let $A$ be the set of elements of $\mathscr{C}\left(L^{2}\right)$ that are not $\mathbf{V}$-inevitable; it is a finite set. For each $g \in A$, choose a complete flow $F_{g}$ on a partial automaton $\mathscr{A}_{g}=\left(Q_{g}, X\right)$ with transition monoid $N_{g} \in \mathbf{V}$ so that, for all $t \in N_{g}$, there is a state $q_{g, t} \in Q_{g}$ so that $\left(q_{g, t} F_{g}, q_{g, t} t F_{g}\right)$ is not stable for $g$. Let $\mathscr{A}=\bigsqcup_{g \in A} \mathscr{A}_{g}$ be the disjoint union of these automata and let $F$ be the flow defined on $\mathscr{A}$ by $\left.F\right|_{\mathscr{A}_{g}}=F_{g}$. Then the transition monoid $N$ of $\mathscr{A}$ is a subdirect product of the $N_{g}$ and so belongs to $\mathbf{V}$. Clearly, $F$ is a complete flow on $\mathscr{A}$. Let $f \in A$ and suppose there exists $t \in N$ so that (4.1) holds for all states $q$ of $\mathscr{A}$. Then, in particular,

$$
q_{f, t} F_{f}=q_{f, t} F \stackrel{\bar{f}}{\rightarrow} q_{f, t} t F=q_{f, t} t F_{f},
$$

a contradiction. This shows that $F$ is the desired complete flow.

Next we introduce the notion of a value for an abstract flow; it will turn out that an abstract flow has a value if and only if it is $\mathbf{V}$-inevitable. 
Definition 4.23 (Values). Let $\mathrm{V}$ be a pseudovariety of monoids. An element $t \in \widehat{F}_{\mathbf{V}}(X)$ is said to be a value of $f \in \mathscr{C}\left(L^{2}\right)$ (relative to $\mathbf{V}$ ) if, for all complete flows $F$ on a partial automaton $\mathscr{A}=(Q, X)$ with transition monoid in $\mathbf{V}$ and all states $q \in Q$, we have

$$
q F \stackrel{\bar{f}}{\rightarrow} q t F .
$$

We use $f v_{\mathbf{V}}$ to denote the set of values of $f$.

Values in the aperiodic setting are very closely related to what are called bases in geometric semigroup theory [20]. A standard compactness argument shows that the $\mathbf{V}$-inevitable flows are exactly those which admit values.

Proposition 4.24. Let $\mathbf{V}$ be a pseudovariety of monoids. Then $f \in \mathscr{C}\left(L^{2}\right)$ is $\mathbf{V}$-inevitable if and only if it has a value in $\widehat{F}_{\mathbf{V}}(X)$.

Proof. Suppose first that $t \in f v_{\mathbf{V}}$ and let $F$ be a complete flow on an automaton $\mathscr{A}=(Q, X)$ with transition monoid $N \in \mathbf{V}$. Then (4.1) holds since (4.2) does.

Conversely, suppose $f$ is $\mathbf{V}$-inevitable. Write $\widehat{F}_{\mathbf{V}}(X)=\lim _{i \in A} V_{i}$, where $\left\{V_{i} \mid\right.$ $i \in A\}$ is the set of all $X$-generated monoids in $\mathbf{V}$. Consider the Cayley graph $\mathscr{V}_{i}=\left(V_{i}, X\right)$ and let $C_{i}$ be the set of all $t \in V_{i}$ so that (4.1) holds for all complete flows $F$ on $\mathscr{V}_{i}$; by Proposition 4.21, $C_{i} \neq \emptyset$. We claim that $\left\{C_{i} \mid i \in A\right\}$ is an inverse subsystem of $\left\{V_{i} \mid i \in A\right\}$. Indeed, suppose that $i \geq j$ and $\pi_{i j}: V_{i} \rightarrow V_{j}$ is the projection. Let $F$ be a complete flow on $\mathscr{V}_{j}$. Then $\pi_{i j} F$ is a complete flow on $\mathscr{V}_{i}$ since $q \pi_{i j} F \stackrel{\bar{x}}{\rightarrow} q \pi_{i j} x F=q x \pi_{i j} F$. Suppose now that $t \in C_{i}$. Let $p \in V_{j}$ and choose a preimage $q \in p \pi_{i j}^{-1}$. Then

$$
p F=q \pi_{i j} F \stackrel{\bar{f}}{\rightarrow}(q t) \pi_{i j} F=\left(p\left(t \pi_{i j}\right)\right) F
$$

and so $t \pi_{i j} \in C_{j}$.

Since an inverse limit of non-empty finite sets is non-empty [30, Lemma 3.1.22] $C=\varliminf_{i \in A} C_{i} \leq \widehat{F}_{\mathbf{V}}(X)$ is non-empty. Choose $t \in C$; we claim that $t$ is a value for $f$. Let $\mathscr{A}=(Q, X)$ be any partial automaton with transition monoid $V_{i}$ in $\mathbf{V}$ and let $F$ be a complete flow on $\mathscr{A}$. Let $q \in Q$. We need to show that $q F \stackrel{\bar{f}}{\rightarrow} q t F$. First we view $F$ as a flow on the complete automaton $\mathscr{A}^{\square}=(Q \cup\{\square\}, X)$ by defining $\square F=B$; note that $V_{i}$ is still the transition monoid of $\mathscr{A}^{\square}$. Next define a complete flow $F^{\prime}$ on $\mathscr{V}_{i}$ by putting $v F^{\prime}=q v F$. To verify that this is a complete flow, note that

$$
v F^{\prime}=q v F \stackrel{\bar{x}}{\rightarrow} q v x F=v x F^{\prime}
$$

since $F$ is a complete flow. Let $\pi_{i}: \widehat{F}_{\mathbf{V}}(X) \rightarrow V_{i}$ be the continuous projection. Then $C \pi_{i} \subseteq C_{i}$ and so if $I$ is the identity of $V$, then

$$
q F=I F^{\prime} \stackrel{\bar{f}}{\rightarrow} I t \pi_{i} F^{\prime}=q t F,
$$

as required. This shows that $C \subseteq f v_{\mathbf{V}}$, completing the proof.

4.3. The values lemma. Here we establish that the $\mathbf{V}$-inevitable flows form a submonoid of $\mathscr{C}\left(L^{2}\right)$ with certain closure properties.

Lemma 4.25 (Values lemma). Let $\mathbf{V}$ be a pseudovariety. The collection $\mathrm{M}_{\mathbf{V}}(L)$ of all elements of $\mathscr{C}\left(L^{2}\right)$ that have values satisfies:

(1) (Identity) The multiplicative identity I of $\mathscr{C}\left(L^{2}\right)$ is in $\mathrm{M}_{\mathbf{V}}(L)$. 
(2) (Points) For all $x \in X$, free flow along $x$ belongs to $\mathrm{M}_{\mathbf{V}}(L)$.

(3) (Products) If $f_{1}, f_{2} \in \mathrm{M}_{\mathbf{V}}(L)$, then $f_{1} f_{2} \in \mathrm{M}_{\mathbf{V}}(L)$.

(4) (Vacuum) If $f \in \mathrm{M}_{\mathbf{V}}(L)$, then $\overleftarrow{f} \in \mathrm{M}_{\mathbf{V}}(L)$

(5) (Aperiodic Action) If $f \in \mathrm{M}_{\mathbf{V}}(L)$ is a $\mathbf{V}$-aperiodic element, then $f^{\omega+*} \in$ $\mathrm{M}_{\mathbf{V}}(L)$.

(6) (Pointlikes) If $A \subseteq \mathrm{M}_{\mathbf{V}}(L)$ is $\mathbf{V}$-pointlike, then $\bigvee A \in \mathrm{M}_{\mathbf{V}}(L)$.

(7) (Stable pairs) If $(A, T)$ is a $\mathbf{V}$-stable pair for $\mathrm{M}_{\mathbf{V}}(L)$, then we have $\bigvee A$. $(\bigvee T)^{*} \in M_{\mathbf{V}}(L)$.

Moreover, $v_{\mathbf{V}}: \mathrm{M}_{\mathbf{V}}(L) \rightarrow \widehat{F}_{\mathbf{V}}(X)$ is a relational morphism of profinite monoids.

Proof. First we verify (Identity). We do this by showing that the empty word $\varepsilon$ is a value for $I$. Indeed, the stable pairs of $I$ are just the elements of the form $(\ell, \ell)$ with $\ell \in L$. So if $\mathscr{A}=(Q, X)$ is any partial automaton, then $q F \stackrel{\bar{I}}{\rightarrow} q F$ for all complete flows $F$ on $\mathscr{A}$.

Next we verify (Points). We claim that $x$ is a value for free flow along $x$. Indeed, by definition of a complete flow on a partial automaton $\mathscr{A}=(Q, X)$, if $q \in Q$, then

$$
q F \stackrel{\bar{x}}{\rightarrow} q x F,
$$

establishing that $x$ is a value for free flow along $x$.

Turning to (Products), we claim that if $u \in f v_{\mathbf{V}}$ and $t \in g v_{\mathbf{V}}$, then $u t \in(f g) v_{\mathbf{V}}$. Indeed, let $F$ be a complete flow on a partial automaton $\mathscr{A}=(Q, X)$ with transition monoid in $\mathbf{V}$. Then, for all $q \in Q$,

$$
q F \stackrel{\bar{f}}{\rightarrow} q u F \stackrel{\bar{g}}{\rightarrow} q u t F .
$$

From this we obtain $q F \stackrel{\overline{f g}}{\rightarrow} q u t F$, as required.

The proofs for (Identity) and (Products) show that $v_{\mathbf{V}}$ is indeed a relational morphism of monoids. But if $t \in \overline{f v_{\mathbf{V}}}$, then in every finite $X$-generated monoid in $\mathbf{V}, t$ agrees with a value of $f$ and so (4.2) holds. Consequently, $t$ is a value for $f$. Thus $f v_{\mathbf{V}}$ is closed and so $v_{\mathbf{V}}$ is a relational morphism of profinite monoids.

Next, we verify (Vacuum). Suppose $f \in \mathrm{M}_{\mathbf{V}}(L)$; we claim that the empty word $\varepsilon$ is a value for $\overleftarrow{f}$. Let $F$ be a complete flow on a partial automaton with transition monoid in V. By Proposition 4.24, $f$ is $\mathbf{V}$-inevitable and hence, by Lemma 4.19, $(q F) \overleftarrow{f}=q F$. Therefore, $(q F, q F)$ is a stable pair for $\overleftarrow{f}$, establishing that $\varepsilon \in \overleftarrow{f} v_{\mathbf{V}}$

To check (Aperiodic Action), suppose that $f \in \mathrm{M}_{\mathbf{V}}(L)$ is $\mathbf{V}$-aperiodic; we show that $f^{\omega+*}$ is $\mathbf{V}$-inevitable and hence has a value. Let $\mathscr{A}=(Q, X)$ be a partial automaton with transition monoid $N \in \mathbf{V}$. Let $\pi: \widehat{F}_{\mathbf{V}}(X) \rightarrow N$ be the canonical projection. Then $v_{\mathbf{V}} \pi: \mathbf{M}_{\mathbf{V}}(L) \rightarrow N$ is a relational morphism and so there exists an aperiodic element $n \in N$ and $t \in f v_{\mathbf{V}}$ so that $t \pi=n$.

Let $F$ be a complete flow on $\mathscr{A}$. We claim that, for all states $q \in Q$, we have $q F \stackrel{\overline{f^{\omega+*}}}{\longrightarrow}\left(q n^{\omega}\right) F$. First note that $t^{\omega}$ is a value for $f^{\omega}$ since $v_{\mathbf{V}}$ is a relational morphism of profinite monoids. Thus $q F \stackrel{\overline{f^{\omega}}}{\longrightarrow} q t^{\omega} F$. Moreover,

$$
q n^{\omega} F \stackrel{\bar{f}}{\rightarrow} q n^{\omega} n F=q n^{\omega} F
$$


since $t$ is a value for $f, n=t \pi$ and $n$ is aperiodic. Thus

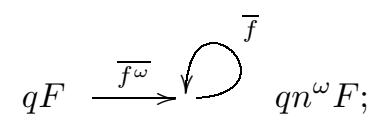

in other words, $q F \stackrel{\overline{f^{\omega+*}}}{\longrightarrow} q n^{\omega} F$. This establishes that $f$ is $\mathbf{V}$-inevitable.

Next suppose that $A \subseteq \mathrm{M}_{\mathbf{V}}(L)$ is $\mathbf{V}$-pointlike. Let $F$ be a complete flow on a partial automaton $\mathscr{A}=(Q, X)$ with transition monoid $N \in \mathbf{V}$. Let $\pi: \widehat{F}_{\mathbf{V}}(X) \rightarrow N$ be the canonical projection. Then $\psi=v_{\mathbf{V}} \pi: \mathrm{M}_{\mathbf{V}}(L) \rightarrow N$ is a relational morphism and so there exists an element $n \in N$ so that $A \subseteq n \psi^{-1}$. Hence, for each $a \in A$, we can find $t_{a} \in a v_{\mathbf{V}}$ so that $t_{a} \pi=n$. Then $q F \stackrel{\bar{a}}{\rightarrow} q n F$ is stable for all $a \in A$ and therefore

$$
q F \stackrel{\overline{\mathrm{V} A}}{\longrightarrow} q n F .
$$

Thus $\bigvee A$ is $\mathbf{V}$-inevitable and so belongs to $\mathrm{M}_{\mathbf{V}}(L)$.

Finally, suppose that $(A, T)$ is a $\mathbf{V}$-stable pair for $\mathbf{M}_{\mathbf{V}}(L)$. As before, let $F$ be a complete flow on a partial automaton $\mathscr{A}=(Q, X)$ with transition monoid $N \in \mathbf{V}$ and denote by $\pi: \widehat{F}_{\mathbf{V}}(X) \rightarrow N$ the canonical projection. Then $\psi=v_{\mathbf{V}} \pi: \mathrm{M}_{\mathbf{V}}(L) \rightarrow$ $N$ is a relational morphism and so there exists an element $n \in N$ so that $A \subseteq n \psi^{-1}$ and $T \subseteq \operatorname{Stab}(n) \psi^{-1}$. It follows that

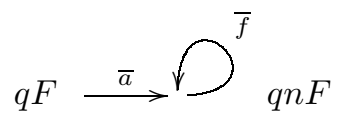

for all $a \in A$ and $f \in T$ and so

$$
q F \stackrel{\overline{\mathrm{V} A \cdot(\mathrm{V} T)^{*}}}{\longrightarrow} q n F .
$$

We conclude that $\bigvee A \cdot(\bigvee T)^{*} \in \mathrm{M}_{\mathbf{V}}(L)$.

4.4. The $n$-flow monoid. To create our lower bound for complexity $n$, we would like to use $\mathbf{M}_{\mathbf{C}_{n-1}}(L)$, but it is not clear that this set is computable. In fact, computability of $\mathbf{C}_{n}$-aperiodic elements for all $n \geq 0$ implies the decidability of complexity by Corollary 4.16. So instead we use the effectively constructible set of $(n-1)$-loopable elements.

Definition 4.26 ( $n$-flow monoid). The $n$-flow monoid $\mathrm{M}_{n}(L)$, for $n \geq 0$, is the smallest subset of $\mathscr{C}\left(L^{2}\right)$ satisfying the following axioms:

(1) (Identity) The multiplicative identity $I$ of $\mathscr{C}\left(L^{2}\right)$ is in $\mathrm{M}_{n}(L)$.

(2) (Points) For all $x \in X$, free flow along $x$ belongs to $\mathrm{M}_{n}(L)$.

(3) (Products) If $f_{1}, f_{2} \in \mathrm{M}_{n}(L)$, then $f_{1} f_{2} \in \mathrm{M}_{n}(L)$.

(4) (Vacuum) If $f \in \mathrm{M}_{n}(L)$, then $\overleftarrow{f} \in \mathrm{M}_{n}(L)$

(5) (Loops) If $f \in \mathrm{M}_{n}(L)$ is $n$-loopable, then $f^{\omega+*} \in \mathrm{M}_{n}(L)$.

We obtain as an immediate corollary of Lemma 4.25 and Proposition 4.13

Corollary 4.27. The set $\mathrm{M}_{n}(L)$ is an effectively computable submonoid of $\mathrm{M}_{\mathbf{C}_{n}}(L)$. Hence each element of $\mathrm{M}_{n}(L)$ has a value relative to $\mathbf{C}_{n}$ and $v_{\mathbf{C}_{n}}: M_{n}(L) \rightarrow$ $\widehat{F}_{\mathbf{C}_{n}}(X)$ is a relational morphism. Consequently, each element of $M_{n}(L)$ is $\mathbf{C}_{n}$ inevitable. 
The proof in fact shows the following: each element of $\mathrm{M}_{0}(L)$ has a value in $F_{\mathbf{A}}^{\omega}(X)$. Thus for complexity one, we are looking at a variation of the notion of tameness as per [2, 3] in the sense that we are cutting down from the free pro-A monoid to an algebraically defined submonoid.

It is natural to ask why we do not choose some of the other properties from Lemma 4.25] in our definition of $M_{n}(L)$. For instance, A-pointlikes are decidable [10, [12, 30, so why not allow them to be joined in the definition of $M_{0}(L)$ ? It turns out that they are not necessary.

Proposition 4.28. Let $A$ be an $\mathbf{A}$-pointlike subset of $M_{0}(L)$. Then there exists $f \in M_{0}(L)$ so that $\bigvee A \leq f$.

Proof. According to [30, Theorem 4.19.2], the collection of A-pointlike subsets of $M_{0}(L)$ is the smallest subsemigroup of $P\left(M_{0}(L)\right)$ containing the singletons and closed under $Z \mapsto Z^{\omega} \bigcup_{k>0} Z^{k}$. Let $C$ be the collection of subsets of $M_{0}(L)$ with an upper bound in $M_{0}(L)$. We show that $C$ satisfies these properties. Clearly all singletons belong to $C$. If $Y, Z \in C$ and $f, g \in M_{0}(L)$ bound $Y$ and $Z$ respectively, then $f g$ bounds each element of $Y Z$ since $M_{0}(L)$ is an ordered monoid. Finally, suppose $f \in M_{0}(L)$ is an upper bound for $Z \in C$. Then each element of $Z^{k}$ is below $f^{k}$, which in turn is below $f^{*}$. Thus $f^{\omega+*}$ is an upper bound for $Z^{\omega} \cup \bigcup_{k \geq 0} Z^{k}$. This completes the proof.

4.5. The $\mathcal{F}_{n}$-operator. We continue to denote $\operatorname{SP}(M, X)$ by $L$. Fix $n \geq 0$. The following definition is crucial to what follows.

Definition $4.29\left(\mathcal{F}_{n}\right.$-operator $)$. Define a closure operator $\mathcal{F}_{n} \in \mathscr{C}(L)$ by

$$
\mathcal{F}_{n}=\bigvee_{f \in M_{n}(L)} \overleftarrow{f}
$$

We sometimes call $\mathcal{F}_{n}$ the vacuum.

The stable set for $\mathcal{F}_{n}$ is the intersection $\bigcap_{f \in \mathrm{M}_{n}(L)} \operatorname{dom} \bar{f}$.

Proposition 4.30. Viewing $\mathcal{F}_{n}$ as a two-variable closure operator, we have that $\mathcal{F}_{n}$ is an idempotent of $\mathrm{M}_{n}(L)$.

Proof. This is immediate from Axiom (Products), Axiom (Vacuum) and Proposition 2.8 .

Thus it is natural to localize $\mathrm{M}_{n}(L)$ at $\mathcal{F}_{n}$, i.e., work with the subsemigroup $\mathcal{F}_{n} \mathrm{M}_{n}(L) \mathcal{F}_{n}$ of $\mathrm{M}_{n}(L)$.

Definition $4.31\left(\mathcal{F}_{n}\right.$-stable). An element $\ell \in L$ is said to be $\mathcal{F}_{n}$-stable if $\ell \mathcal{F}_{n}=\ell$. An element $f$ of $\mathrm{M}_{n}(L)$ is said to be $\mathcal{F}_{n}$-stable if $\mathcal{F}_{n} f \mathcal{F}_{n}=f$.

Our first example of an $\mathcal{F}_{n}$-stable subset is the bottom.

Proposition 4.32. The bottom $B$ of $L$ is $\mathcal{F}_{n}$-stable.

Proof. Proposition 4.20 implies $B=B \overleftarrow{\mathcal{F}_{n}}=B \mathcal{F}_{n}$.

Our next observation is that any flow on an automaton of complexity at most $n$ takes on only $\mathcal{F}_{n}$-stable values. More precisely, we have:

Lemma 4.33. Let $F$ be a complete flow on a partial automaton $\mathscr{A}=(Q, X)$ with transition monoid in $\mathbf{C}_{n}$. Then $q F$ is $\mathcal{F}_{n}$-stable for all $q \in Q$. 
Proof. Since each element of $\mathrm{M}_{n}(L)$ is $\mathbf{C}_{n}$-inevitable by Corollary 4.27 and $\mathcal{F}_{n}=\overleftarrow{\mathcal{F}_{n}}$ (cf. Remark 2.15), it follows that $(q F) \mathcal{F}_{n}=q F$ by Lemma 4.19 .

As a consequence, we can prove that sets do not change under $\mathcal{F}_{n}$, and hence the points of $\mathrm{SP}(M, X)$ are $\mathcal{F}_{n}$-stable.

Proposition 4.34. If $Y \subseteq R$, then $(Y,\{Y\})$ is $\mathcal{F}_{n}$-stable. In particular, if $r \in R$, then $(r, r)$ is $\mathcal{F}_{n}$-stable.

Proof. Let $\mathscr{A}$ be the complete automaton

$$
q_{0} \stackrel{x}{\longrightarrow} q_{1}^{X}
$$

where an edge labelled $X$ is shorthand for a set of edges labelled by each element of $X$. Consider the complete flow $F$ on $\mathscr{A}$ given by $q_{0} F=(Y,\{Y\})$ and $q_{1} F=$ $(R,\{R\})$. This is a flow since, for any $x \in X$, trivially $Y x \subseteq R$ and any partial map $Y /\{Y\} \rightarrow R /\{R\}$ is injective. Of course, $(R,\{R\})$ is stable for $x^{*}$ all $x \in X$ being the top of $\mathrm{SP}(M, X)$. Since $\mathscr{A}$ has aperiodic transition monoid, it follows $(Y,\{Y\}) \mathcal{F}_{n}=(Y,\{Y\})$ by Lemma 4.33

Thus the vacuum only changes partitions and not sets.

Corollary 4.35. If $(Y, P) \in L$, then $(Y, P) \mathcal{F}_{n}=\left(Y, P^{\prime}\right)$ for some partition $P^{\prime}$ on $Y$.

Proof. Since $(Y, P) \leq(Y,\{Y\})$, we have

$$
(Y, P) \leq(Y, P) \mathcal{F}_{n} \leq(Y,\{Y\}) \mathcal{F}_{n}=(Y,\{Y\})
$$

by Proposition 4.34. Thus $(Y, P) \mathcal{F}_{n}=\left(Y, P^{\prime}\right)$ for some partition $P^{\prime}$.

The operator $\mathcal{F}_{n}$ captures the back-flow from all elements of $\mathrm{M}_{n}(L)$. More precisely we have the following proposition.

Proposition 4.36. Suppose $\ell \in L$ is $\mathcal{F}_{n}$-stable and let $f \in \mathrm{M}_{n}(L)$. Then $\ell \overleftarrow{f}=\ell$ that is, $\ell \stackrel{f}{\rightarrow} B=(\ell, \ell \vec{f})$.

Proof. This follows since $\overleftarrow{f} \leq \mathcal{F}_{n}$, and hence the fact that $\ell$ is $\mathcal{F}_{n}$-stable implies that $\ell$ is stable for $\overleftarrow{f}$

Proposition 4.37. The map $\mathscr{C}\left(L^{2}\right) \rightarrow \mathscr{C}\left(L^{2}\right)$ given by $f \mapsto \mathcal{F}_{n} f \mathcal{F}_{n}$ is a closure operator satisfying

$$
\mathcal{F}_{n} f g \mathcal{F}_{n} \leq\left(\mathcal{F}_{n} f \mathcal{F}_{n}\right)\left(\mathcal{F}_{n} g \mathcal{F}_{n}\right) .
$$

The set $\mathcal{F}_{n} \mathrm{M}_{n}(L) \mathcal{F}_{n}$ of $\mathcal{F}_{n}$-stable elements of $\mathrm{M}_{n}(L)$ is a subsemigroup with identity $\mathcal{F}_{n}$. Moreover, an element $f \in \mathrm{M}_{n}(L)$ is $\mathcal{F}_{n}$-stable if and only if its stable pairs belong to $L \mathcal{F}_{n} \times L \mathcal{F}_{n}$.

Proof. Proposition 2.10 implies $f \mapsto \mathcal{F}_{n} f \mathcal{F}_{n}$ is a closure operator. Equation (4.4) is an immediate consequence of the inequality $1_{L} \leq \mathcal{F}_{n}$. It is obvious that $\mathcal{F}_{n} \mathrm{M}_{n}(L) \mathcal{F}_{n}$ is a subsemigroup with identity $\mathcal{F}_{n}$. Since as a binary relation, $\mathcal{F}_{n}=1_{L \mathcal{F}_{n}}$, the final statement is clear.

Recall that if $P$ is a partially ordered set, a subset $K \subseteq P$ is called a filter if $k \in K$ and $p \geq k$ imply $p \in K$. 
Corollary 4.38. The subset $\mathcal{F}_{n} \mathrm{M}_{n}(L) \mathcal{F}_{n}$ is a filter in $\mathrm{M}_{n}(L)$. In particular, if $f \in \mathcal{F}_{n} \mathrm{M}_{n}(L) \mathcal{F}_{n}$ is n-loopable, then $f^{\omega+*} \in \mathcal{F}_{n} \mathrm{M}_{n}(L) \mathcal{F}_{n}$.

Proof. Suppose that $f \in \mathrm{M}_{n}(L)$ is $\mathcal{F}_{n}$-stable and $g \geq f$. Then Propositions 2.3 and 4.37 show that

$$
\operatorname{Im} g \subseteq \operatorname{Im} f \subseteq L \mathcal{F}_{n} \times L \mathcal{F}_{n}
$$

Another application of Proposition 4.37 lets us deduce that $g$ is $\mathcal{F}_{n}$-stable.

The last statement follows since $f^{\omega}$ is $\mathcal{F}_{n^{-}}$stable and $f^{\omega} \leq f^{\omega+*}$ by Proposition 2.10

Definition $4.39\left(\mathcal{F}_{n}\right.$-stable transformation monoid). There is a monoid action of $\mathcal{F}_{n} \mathrm{M}_{n}(L) \mathcal{F}_{n}$ on $L \mathcal{F}_{n}$ by total functions defined by $\ell \cdot f=\ell \vec{f}$ for $\ell \in L \mathcal{F}_{n}$, $f \in \mathcal{F}_{n} \mathrm{M}_{n}(L) \mathcal{F}_{n}$.

Proposition 4.40. The action in Definition 4.39 is well defined.

Proof. Let $\ell \in L \mathcal{F}_{n}$ and $f \in \mathcal{F}_{n} \mathrm{M}_{n}(L) \mathcal{F}_{n}$. Then by the last statement of Proposition 4.37, $\ell \vec{f}$ is $\mathcal{F}_{n}$-closed. It now follows from Propositions 2.17 and 4.36 that the action is a well-defined semigroup action. Clearly $\mathcal{F}_{n}$ acts as the identity on $L \mathcal{F}_{n}$ since $\overrightarrow{\mathcal{F}_{n}}=\mathcal{F}_{n}$ by Remark 2.15

The action of $\mathcal{F}_{n} \mathrm{M}_{n}(L) \mathcal{F}_{n}$ is not faithful. In fact, we have:

Proposition 4.41. Suppose that $g \in \mathcal{F}_{n} \mathrm{M}_{n}(L) \mathcal{F}_{n}$ is n-loopable. Then the equality $\ell \cdot g^{\omega+*}=\ell \cdot g^{\omega+*} g^{\omega}$ holds for any $\ell \in L \mathcal{F}_{n}$.

Proof. Observe that $\ell \cdot g^{\omega+*} g^{\omega}=\ell \cdot g^{\omega+*} g^{\omega} g$ by Lemma 2.41, So if $\ell_{1}=\ell \cdot g^{\omega+*} g^{\omega}$, then $\ell_{1} \cdot g=\ell_{1}$. Therefore, we have

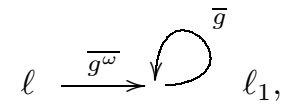

that is, $\ell \overline{g^{\omega+*}} \ell_{1}$. We conclude that $\ell \cdot g^{\omega+*} \leq \ell_{1}$ by definition of forward-flow. But $g^{\omega+*} g^{\omega} \leq g^{\omega+*}$ by Lemma 2.40, so $\ell_{1} \leq \ell \cdot g^{\omega+*}$. This completes the proof.

\section{THE LOWER BOUND}

This section constructs our lower bound for complexity. More precisely, given a finite $X$-generated group mapping monoid $M$ with distinguished $\mathscr{R}$-class $R$, we shall effectively construct a collection of $\mathbf{C}_{n}$-inevitable elements of $\operatorname{SP}(M, X)$, for $n \geq 0$. Then the results of Section 3 show that a necessary condition for $M$ to have complexity $n+1$ is that no element of the form $(Y, P)$ of this collection have a block $B$ of $P$ containing distinct $\mathscr{H}$-equivalent elements of $R$; cf. Corollary 3.10 .

5.1. The evaluation monoid. We continue to denote $\operatorname{SP}(M, X)$ by $L$ and fix $n \geq 0$. The evaluation transformation semigroup will be the combinatorial object that encodes the $\mathbf{C}_{n}$-inevitable elements of $L$ as well as an action of a certain submonoid of $\mathcal{F}_{n} \mathrm{M}_{n}(L) \mathcal{F}_{n}$ on these elements.

First we need the following notion of a well-formed formula.

Definition 5.1 (Well-formed formulae). Let $X$ be an alphabet. We define a wellformed formula inductively as follows. The empty string $\varepsilon$ is a well-formed formula. Each letter $x \in X$ is a well-formed formula. If $\tau, \sigma$ are well-formed formulae, then so is $\tau \sigma$. If $\tau$ is a well-formed formula that is not a proper power (i.e., of the form 
$(\sigma)^{n}$ where $\left.n>1\right)$, then $\tau^{\omega+*}$ is also a well-formed formula. The set of well-formed formulae is denoted by $\Omega(X)$. Well-formed formulae will be denoted by Greek letters. As a convention, if $\tau=\sigma^{n}$, where $\sigma$ is not a proper power, then we set $\tau^{\omega+*}=\sigma^{\omega+*}$; in other words, we extract roots before applying the unary operation.

We want to interpret well-formed formulae in $\mathcal{F}_{n} \mathrm{M}_{n}(L) \mathcal{F}_{n}$.

Definition 5.2 (Standard Interpretation). Define recursively a partial function $\Upsilon: \Omega(X) \rightarrow \mathcal{F}_{n} \mathrm{M}_{n}(L) \mathcal{F}_{n}$ as follows. Set $\varepsilon \Upsilon=\mathcal{F}_{n}$ and $x \Upsilon=\mathcal{F}_{n} x \mathcal{F}_{n}$ for $x \in X$. If $\Upsilon$ is already defined on $\tau, \sigma \in \Omega(X)$, set $(\tau \sigma) \Upsilon=\tau \Upsilon \sigma \Upsilon$. If $\tau \in \Omega(X)$ is not a proper power and $\tau \Upsilon$ is defined and $n$-loopable, set $\tau^{\omega+*} \Upsilon=(\tau \Upsilon)^{\omega+*}$. We normally omit $\Upsilon$ and assume that $\tau \in \Omega(X)$ is being evaluated in $\mathcal{F}_{n} \mathrm{M}_{n}(L) \mathcal{F}_{n}$ according to the standard interpretation. However, when there is danger of confusion with free flow, we use $\Upsilon$.

We can now define the set of $\mathcal{F}_{n}$-states.

Definition $5.3\left(\mathcal{F}_{n}\right.$-states $)$. The collection of $\mathcal{F}_{n}$-states is by definition the smallest

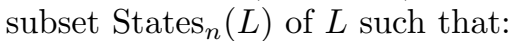

(1) (Points) $(r, r) \in \operatorname{States}_{n}(L)$, for all $r \in R$;

(2) (Forward-flow) $\ell \vec{\tau} \in \operatorname{States}_{n}(L)$ for all $\ell \in \operatorname{States}_{n}(L), \tau \in \Omega(X)$;

(3) (Order ideal) if $\ell \leq \ell^{\prime} \in \operatorname{States}_{n}(L)$, then $\ell \mathcal{F}_{n} \in \operatorname{States}_{n}(L)$.

Remark 5.4. If there exists $(Y, P) \in \operatorname{States}_{n}(L)$ and $r \neq s \in Y$ such that $r P s$, then $(\{r, s\},\{\{r, s\}\}) \in \operatorname{States}_{n}(L)$ by Axiom (Order Ideal) since $(\{r, s\},\{\{r, s\}\})$ is $\mathcal{F}_{n}$-stable by Proposition 4.34 .

First we prove that $\mathcal{F}_{n}$-states are $\mathcal{F}_{n}$-stable.

Proposition 5.5. The elements of $\operatorname{States}_{n}(L)$ are $\mathcal{F}_{n}$-stable.

Proof. We show $L \mathcal{F}_{n}$ satisfies the axioms of Definition 5.3. Axiom (Points) holds by Proposition 4.34. Since the front of any stable pair of an element of $\mathcal{F}_{n} \mathrm{M}_{n}(L) \mathcal{F}_{n}$ is $\mathcal{F}_{n}$-stable by Proposition 4.37. Axiom (Forward-flow) holds for $L \mathcal{F}_{n}$. Axiom (Order ideal) trivially holds for $L \mathcal{F}_{n}$. This establishes $\operatorname{States}_{n}(L) \subseteq L \mathcal{F}_{n}$.

We do not have any axiom about back-flow since if $f \in M_{n}(L)$ is $\mathcal{F}_{n}$-stable and $\ell$ is an $\mathcal{F}_{n}$-state, then $(\ell, B) f=(\ell, \ell \vec{f})$, so there is no back-flow.

Definition 5.6 (Evaluation transformation monoid). The action, from Definition 4.39, of $\mathcal{F}_{n} \mathrm{M}_{n}(L) \mathcal{F}_{n}$ on $L \mathcal{F}_{n}$ restricts to an action of $\Omega(X) \Upsilon$ on $\operatorname{States}_{n}(L)$ by Axiom (Forward-flow). The associated faithful transformation monoid is denoted by

$$
\mathscr{E}_{n}(L)=\left(\operatorname{States}_{n}(L), M\left(\mathscr{E}_{n}(L)\right)\right)
$$

and called the evaluation transformation monoid. We term $M\left(\mathscr{E}_{n}(L)\right)$ the evaluation monoid.

5.2. Action on sets. In this section, we try to understand how $\Omega(X)$ acts on set-partitions in the set coordinate. For this reason, we will interpret elements of $\Omega(X)$ as elements of $\mathscr{C}\left(\mathrm{S}(M, X)^{2}\right)$, as well.

Definition 5.7 (Interpretation on sets). Define $\Psi: \Omega(X) \rightarrow \mathscr{C}\left(\mathrm{S}(M, X)^{2}\right)$ as follows. Set $\varepsilon \Psi$ to be the identity of $\mathscr{C}\left(\mathrm{S}(M, X)^{2}\right)$. If $x \in X$, then $x \Psi$ is free flow along $x$. If $\sigma, \tau \in \Omega(x)$, then $(\sigma \tau) \Psi=\sigma \Psi \tau \Psi$. If $\sigma$ is not a proper power, then $\sigma^{\omega+*} \Psi=\sigma \Psi^{\omega+*}$. Again, we drop $\Psi$ from the notation when no confusion can arise. 
Our aim is to establish a compatibility between the interpretation of $\Omega(X)$ in the set flow lattice and the set-partition flow lattice.

Proposition 5.8. Let $\tau \in \Omega(X)$ be in $\operatorname{dom} \Upsilon$. Then $(Y, P) \stackrel{\bar{\tau}}{\rightarrow}\left(Y^{\prime}, P^{\prime}\right)$ implies $Y \stackrel{\bar{\tau}}{\rightarrow} Y^{\prime}$.

Proof. We go by induction on the recursive construction of well-formed formulae. If $(Y, P) \stackrel{\overline{\mathcal{F}_{n}}}{\longrightarrow}\left(Y^{\prime}, P^{\prime}\right)$, then $Y=Y^{\prime}$ and so $Y \stackrel{\bar{\varepsilon}}{\rightarrow} Y=Y^{\prime}$. If $x \in X$ and $(Y, P) \stackrel{\overline{\mathcal{F}_{n} x \mathcal{F}_{n}}}{\longrightarrow}$ $\left(Y^{\prime}, P^{\prime}\right)$, then $(Y, P) \stackrel{\bar{x}}{\rightarrow}\left(Y^{\prime}, P^{\prime}\right)$ and so $Y x \subseteq Y^{\prime}$. But this says exactly that $Y \stackrel{\bar{x}}{\rightarrow} Y^{\prime}$.

Assume that the desired implication holds for $\sigma, \tau \in \Omega(X)$ and suppose $(Y, P)$ $\stackrel{\overline{\sigma \tau}}{\longrightarrow}\left(Y^{\prime}, P^{\prime}\right)$. Then we can find $\left(Y^{\prime \prime}, P^{\prime \prime}\right)$ so that

$$
(Y, P) \stackrel{\bar{\sigma}}{\rightarrow}\left(Y^{\prime \prime}, P^{\prime \prime}\right) \stackrel{\bar{\tau}}{\rightarrow}\left(Y^{\prime}, P^{\prime}\right) .
$$

Thus by hypothesis we have

$$
Y \stackrel{\bar{\sigma}}{\rightarrow} Y^{\prime \prime} \stackrel{\bar{\tau}}{\rightarrow} Y^{\prime}
$$

and so $Y \stackrel{\overline{\sigma \tau}}{\longrightarrow} Y^{\prime}$, as required. Finally, suppose that $\sigma$ satisfies the conclusion of the proposition, $\sigma \Upsilon$ is $n$-loopable and $\sigma$ is not a proper power. Choose $m$ so that $f^{m}=f^{\omega}$ for all $f \in \mathrm{M}_{n}(\operatorname{SP}(M, X))$ and all $f \in \mathscr{C}\left(\mathrm{S}(M, X)^{2}\right)$. Suppose that $(Y, P) \stackrel{\overline{\sigma^{\omega+*}}}{\longrightarrow}\left(Y^{\prime}, P^{\prime}\right)$. Then

$$
(Y, P) \stackrel{\overline{\sigma^{m}}}{\longrightarrow} \bigcap^{\bar{\sigma}}\left(Y^{\prime}, P^{\prime}\right)
$$

and so by assumption on $\sigma$ and the case of products handled above it follows that

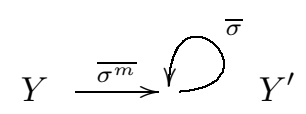

and hence $Y \stackrel{\overline{\sigma^{\omega+*}}}{\longrightarrow} Y^{\prime}$. This completes the proof.

As a corollary, we see that sets only flow forward: there is no back-flow.

Corollary 5.9. If $Y \in \mathrm{S}(M, X)$ and $\tau \in \Omega(X)$, then $Y \overleftarrow{\tau}=Y$.

Proof. Note that $\Upsilon$ is total for $n=0$. Since $\tau \Upsilon \in \mathcal{F}_{0} \mathrm{M}_{0}(\operatorname{SP}(M, X)) \mathcal{F}_{0}$ and $(Y,\{Y\})$ is $\mathcal{F}_{0}$-stable by Proposition 4.34 , it follows that $(Y,\{Y\}) \stackrel{\bar{\tau}}{\rightarrow}(Y,\{Y\}) \vec{\tau}$. Thus $Y \in \operatorname{dom} \tau$ by Proposition 5.8 and hence $Y \overleftarrow{\tau}=Y$

Our next goal is to prove that certain set flows yield set-partition flows.

Proposition 5.10. Let $\tau \in \Omega(X)$. Then, for $Y, Y^{\prime} \in \mathrm{S}(M, X)$, we have $Y \stackrel{\bar{\tau}}{\rightarrow} Y^{\prime}$ if and only if $(Y,\{Y\}) \stackrel{\bar{\tau}}{\rightarrow}\left(Y^{\prime},\left\{Y^{\prime}\right\}\right)$ for $\tau \in \operatorname{dom} \Upsilon$.

Proof. The implication from right to left is a consequence of Proposition 5.8, so we handle the forward implication. Again, we proceed by induction on the recursive definition of well-formed formulae. If $Y \stackrel{\bar{\varepsilon}}{\rightarrow} Y^{\prime}$, then $Y=Y^{\prime}$. Since $(Y,\{Y\})$ is $\mathcal{F}_{n^{-}}$ stable, by Proposition 4.34, it follows that $(Y,\{Y\}) \stackrel{\overline{\mathcal{F}_{n}}}{\longrightarrow}(Y,\{Y\})$. Next, suppose 
$x \in X$ and that $Y \stackrel{\bar{x}}{\rightarrow} Y^{\prime}$. Then $Y x \subseteq Y^{\prime}$. Since any partial function $Y /\{Y\} \rightarrow$ $Y^{\prime} /\left\{Y^{\prime}\right\}$ is injective and $(Y,\{Y\}),\left(Y^{\prime},\left\{Y^{\prime}\right\}\right)$ are $\mathcal{F}_{n}$-stable by Proposition 4.34, we conclude that

$$
(Y,\{Y\}) \stackrel{\overline{\mathcal{F}_{n} x \mathcal{F}_{n}}}{\longrightarrow}\left(Y^{\prime},\left\{Y^{\prime}\right\}\right) .
$$

Assume the proposition holds for $\sigma, \tau \in \Omega(X)$ and suppose $Y \stackrel{\overline{\sigma \tau}}{\longrightarrow} Y^{\prime}$. Then there exists $Y^{\prime \prime}$ so that

$$
Y \stackrel{\bar{\sigma}}{\rightarrow} Y^{\prime \prime} \stackrel{\bar{\tau}}{\rightarrow} Y^{\prime}
$$

By assumption, we have

$$
(Y,\{Y\}) \stackrel{\bar{\sigma}}{\rightarrow}\left(Y^{\prime \prime},\left\{Y^{\prime \prime}\right\}\right) \stackrel{\bar{\tau}}{\rightarrow}\left(Y^{\prime},\left\{Y^{\prime}\right\}\right)
$$

and so $(Y,\{Y\}) \stackrel{\overline{\sigma \tau}}{\longrightarrow}\left(Y^{\prime},\left\{Y^{\prime}\right\}\right)$.

Finally, suppose that the desired conclusion holds for $\sigma \in \Omega(X)$, where $\sigma$ is not a proper power and $\sigma \Upsilon$ is $n$-loopable. Choose $m$ so that $f^{m}=f^{\omega}$ for all $f \in \mathrm{M}_{n}(\mathrm{SP}(M, X))$ and all $f \in \mathscr{C}\left(\mathrm{S}(M, X)^{2}\right)$. Then the proposition also holds for $\sigma^{m}$. Therefore, if $Y \stackrel{\overrightarrow{\sigma^{\omega+*}}}{\longrightarrow} Y^{\prime}$, then

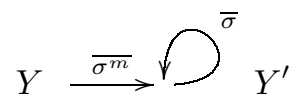

and hence

$$
(Y,\{Y\}) \stackrel{\overline{\sigma^{m}}}{\longrightarrow} \bigcap^{\bar{\sigma}}\left(Y^{\prime},\left\{Y^{\prime}\right\}\right)
$$

by the induction hypothesis. We conclude that $(Y,\{Y\}) \stackrel{\overline{\sigma^{\omega+*}}}{\longrightarrow}\left(Y^{\prime},\left\{Y^{\prime}\right\}\right)$, as required.

As a corollary, we can determine what happens to the sets when applying elements of $\Omega(X)$ to elements of $\operatorname{SP}(M, X)$.

Corollary 5.11. Suppose $\tau \in \Omega(X)$ belongs to $\operatorname{dom} \Upsilon$ and that $Y \stackrel{\tau}{\rightarrow} Y^{\prime}=\left(Z, Z^{\prime}\right)$. Then, for any partitions $P, P^{\prime}$ on $Y, Y^{\prime}$, respectively, we have

$$
(Y, P) \stackrel{\tau}{\rightarrow}\left(Y^{\prime}, P^{\prime}\right)=\left((Z, Q),\left(Z^{\prime}, Q^{\prime}\right)\right)
$$

for some partitions $Q, Q^{\prime}$ on $Z, Z^{\prime}$, respectively.

Proof. Suppose that $(Y, P) \stackrel{\tau}{\rightarrow}\left(Y^{\prime}, P^{\prime}\right)=\left((W, S),\left(W^{\prime}, S^{\prime}\right)\right)$. Then $W \stackrel{\bar{\tau}}{\rightarrow} W^{\prime}$ by Proposition 5.8. Since $Y \leq W$ and $Y^{\prime} \leq W^{\prime}$, it follows that

$$
\left(Z, Z^{\prime}\right)=Y \stackrel{\tau}{\rightarrow} Y^{\prime} \leq W \stackrel{\tau}{\rightarrow} W^{\prime}=\left(W, W^{\prime}\right) .
$$

On the other hand, $(Z,\{Z\}) \stackrel{\bar{\tau}}{\rightarrow}\left(Z^{\prime},\left\{Z^{\prime}\right\}\right)$ by Proposition 5.10. From the inequalities $(Y, P) \leq(Z,\{Z\})$ and $\left(Y^{\prime}, P^{\prime}\right) \leq\left(Z^{\prime},\left\{Z^{\prime}\right\}\right)$, we must have $(W, S) \leq(Z,\{Z\})$ and $\left(W^{\prime}, S^{\prime}\right) \leq\left(Z^{\prime},\left\{Z^{\prime}\right\}\right)$. Thus $W=Z$ and $W^{\prime}=Z^{\prime}$, as required.

Note that we can define an action of $\Omega(X)$ on subsets of $R$ by setting $Y \cdot \tau=Y \vec{\tau}$. This is an action by Corollary 5.9 and Proposition 2.17. As a consequence of Corollary [5.11] we obtain the following result.

Theorem 5.12. Let $(Y, P)$ be $\mathcal{F}_{n}$-stable and suppose that $\tau \in \Omega(X)$ is in $\operatorname{dom} \Upsilon$. Then $(Y, P) \cdot \tau=\left(Y \cdot \tau, P^{\prime}\right)$ for some partition $P^{\prime}$ on $Y \cdot \tau$. 
Proof. Since $Y \stackrel{\tau}{\rightarrow} B=(Y \overleftarrow{\tau}, Y \vec{\tau})$, Corollary [5.11] guarantees that

$$
(Y, P) \stackrel{\tau}{\rightarrow} B=\left((Y \overleftarrow{\tau}, Q),\left(Y \vec{\tau}, P^{\prime}\right)\right)
$$

for some partitions $Q$ and $P^{\prime}$ (actually $Y=Y^{\overleftarrow{\tau}}$ and $Q=P$ since there is no back-flow on $\mathcal{F}_{n}$-stable set-partitions).

We would like to make a conjecture on what $Y \cdot \tau$ is for $\tau \in \Omega(X)$.

Definition 5.13 (Interpretation in $P(M)$ ). Define a map $\Lambda: \Omega(X) \rightarrow P(M)$ recursively as follows. Set $\varepsilon \Lambda=\{I\}$, where $I$ is the identity of $M$. Put $x \Lambda=\left\{[x]_{M}\right\}$. If $\Lambda$ is defined on $\sigma, \tau$, then set $(\sigma \tau) \Lambda=\sigma \Lambda \tau \Lambda$. If $\sigma$ is not a proper power, put $\sigma^{\omega+*} \Lambda=\bigcup_{k \geq 0}(\sigma \Lambda)^{\omega}(\sigma \Lambda)^{k}$.

Conjecture 5.14. If $\tau \in \Omega(X)$ and $Y \subseteq R$, then $Y \cdot \tau=Y(\tau \Lambda)$.

We do know exactly what happens for strings; things are more complicated for higher rank elements of $\Omega(X)$.

Proposition 5.15. Let $w \in X^{*} \subseteq \Omega(X)$ and suppose that $(Y, P)$ is $\mathcal{F}_{n}$-stable. Let $P=\left\{B_{1}, \ldots, B_{r}\right\}$, where $B_{1}, \ldots, B_{k}$ are the blocks of $P$ with $B_{i} w \neq \emptyset$. Then $B_{1} w, \ldots, B_{k} w$ are disjoint and the equality

$$
(Y, P) \cdot w \Upsilon=\left(Y w,\left\{B_{1} w, \ldots, B_{k} w\right\}\right)=(Y, P) \vec{w}
$$

holds.

Proof. We proceed by induction on $|w|$. If $|w|=0$, then there is nothing to prove. Suppose it is true for $u$ and let $w=u x$ with $x \in X$. Suppose that $B_{1}, \ldots, B_{m}$ are the blocks with $B_{i} u \neq \emptyset$. Then $k \leq m$. By induction,

$$
(Y, P) \cdot u \Upsilon=\left(Y u,\left\{B_{1} u, \ldots, B_{m} u\right\}\right)=(Z, Q) .
$$

Since $(Y, P) \overrightarrow{u \Upsilon}=(Z, Q)$ is $\mathcal{F}_{n}$-stable, Proposition 4.36 yields $(Z, Q) \overleftarrow{x}=(Z, Q)$ Consequently, Proposition 2.27 implies that

$$
(Z, Q) \vec{x}=\left(Y w,\left\{B_{1} w, \ldots, B_{k} w\right\}\right)=(W, S) .
$$

As $x \leq \mathcal{F}_{n} x \mathcal{F}_{n}$, it follows that if $\left(W^{\prime}, S^{\prime}\right)=(Z, Q) \cdot x \Upsilon$, then we have $(W, S) \leq$ $\left(W^{\prime}, S^{\prime}\right)$. Now $W^{\prime}=Z x=Y w$ by Theorem $\left[5.12\right.$ and so $\left(W^{\prime}, S^{\prime}\right)=\left(Z x, S^{\prime}\right)$. But if $(W, S)<\left(W, S^{\prime}\right)$, then two blocks $B_{i} u x, B_{j} u x$ with $i \neq j$ must be contained in a single block of $S^{\prime}$, contradicting $(Z, Q) \stackrel{\bar{x}}{\rightarrow}\left(W, S^{\prime}\right)$. Thus $(W, S)=\left(W, S^{\prime}\right)$, as required.

As $N=\left\langle\mathcal{F}_{n} x \mathcal{F}_{n} \mid x \in X\right\rangle$ is $X$-generated and acts on $\operatorname{States}_{n}(L)$, it is natural to try and compute complexity using $\mathscr{A}=\left(\operatorname{States}_{n}(L), X\right)$, where the transitions come via the action of $N$ on $\operatorname{States}_{n}(L)$. One could then define a complete flow $F$ on $\mathscr{A}$ by $(Y, P) F=(Y, P)$. If $N$ had complexity at most $n$, then this would prove that $\operatorname{States}_{n}(L)$ contains all maximal $\mathbf{C}_{n}$-inevitable elements. Unfortunately, $M \cong N$ and so we do not know the complexity of $N$.

Proposition 5.16. The submonoid $\left\langle\mathcal{F}_{n} x \mathcal{F}_{n} \mid x \in X\right\rangle$ of $M\left(\mathscr{E}_{n}(L)\right)$ is isomorphic to $M$.

Proof. If $r \in R$, then $(r, r) \in \operatorname{States}_{n}(L)$ and Proposition 5.15 readily implies, for $w \in X^{*}$, that $(r, r) \cdot w \Upsilon=(r w, r w)$ (where as usual $r w=\emptyset$ if it is not defined). Since $M$ acts faithfully on $R$, it follows that $v \Upsilon=w \Upsilon$ implies $[v]_{M}=[w]_{M}$ for $v, w \in X^{*}$. Conversely, Proposition 5.15 immediately yields that $[v]_{M}=[w]_{M}$ implies $v \Upsilon=w \Upsilon$ for $v, w \in X^{*}$. This completes the proof. 
5.3. The lower bound theorem. We are now ready to prove the lower bound theorem for complexity. Once again $M$ is a fixed group mapping monoid generated by $X$ and we set $L=\mathrm{SP}(M, X)$.

Theorem 5.17 (The Inevitability Theorem). Each element of $\operatorname{States}_{n}(L)$ is $\mathbf{C}_{n^{-}}$ inevitable.

Proof. Let $\mathscr{I}$ be the set of $\mathbf{C}_{n}$-inevitable elements of $L$. We show that it satisfies the axioms of Definition [5.3. This will show that each element of $\operatorname{States}_{n}(L)$ is $\mathbf{C}_{n}$-inevitable.

As was observed earlier, points are $\mathbf{V}$-inevitable for any pseudovariety $\mathbf{V}$. Suppose that $\ell \in \mathscr{I}$ and $f \in \mathrm{M}_{n}(L)$. We show that $\ell \vec{f} \in \mathscr{I}$. This will imply Axiom (Forward-flow). If $\ell \vec{f}=B$, there is nothing to prove. So assume henceforth that $\vec{\ell} \neq B$. Let $\mathscr{A}=(Q, X)$ be any partial automaton with transition monoid in $\mathbf{C}_{n}$ and suppose $F$ is a complete flow on $\mathscr{A}$. By Corollary 4.27, there is an element $t \in f v_{\mathbf{C}_{n}}$. Since $\ell$ is $\mathbf{C}_{n}$-inevitable, there is a state $q \in Q$ such that $\ell \leq q F$. By the definition of a value, $q F \stackrel{\bar{f}}{\rightarrow} q t F$. Since $(\ell, B) \leq(q F, q t F)$, it follows that

$$
\ell \stackrel{f}{\rightarrow} B=(\ell \overleftarrow{f}, \ell \vec{f}) \leq(q F, q t F)
$$

in particular, $q t \neq \square$ as $\ell \vec{f} \neq B$. Since $\mathscr{A}$ and $F$ were arbitrary, we deduce that $\ell \vec{f} \in \mathscr{I}$.

For Axiom (Order ideal), suppose that $\ell^{\prime} \in \mathscr{I}$ and $\ell \leq \ell^{\prime}$. Since the $\mathbf{C}_{n^{-}}$ inevitable elements of $L$ form an order ideal, it follows that $\ell \in \mathscr{I}$. So let $F$ be a complete flow on a partial automaton $\mathscr{A}$ with transition monoid in $\mathbf{C}_{n}$. Then $\ell \leq q F$ for some state $q$. But $q F$ is $\mathcal{F}_{n}$-stable by Lemma 4.33. Therefore, $\ell \mathcal{F}_{n} \leq q F$ establishing that $\ell \mathcal{F}_{n} \in \mathscr{I}$.

This leads to our lower bound for complexity, which is the main result of this paper.

Theorem 5.18 (Lower Bound Theorem for Complexity). Suppose $M$ is a finite $X$-generated group mapping monoid with distinguished $\mathscr{R}$-class $R$. If there exists $r \neq s \in R$ such that $r \mathscr{H} s$ and

$$
(\{r, s\},\{\{r, s\}\}) \in \operatorname{States}_{n}(\operatorname{SP}(M, X)),
$$

then $M$ has complexity at least $n+2$.

Proof. This follows directly from Theorem 5.17, Corollary 3.10 and Remark 5.4,

We remark that the proof of Theorem 5.17 would seem to indicate that in Axiom (Forward-flow) we should allow any element of $\mathcal{F}_{n} \mathrm{M}_{n}(L) \mathcal{F}_{n}$ to be used. But it follows easily from the definition of $\mathrm{M}_{n}(L)$, from the fact that $\mathcal{F}_{n}$ is a closure operator and from (4.4) that each element of $\mathrm{M}_{n}(L)$ is below an element determined by the standard interpretation of a well-formed formula. More precisely, the set of elements of $\mathscr{C}\left(L^{2}\right)$ that are below the standard interpretation of a well-formed formula satisfies the axioms of Definition 4.26. Hence we would obtain no new maximal $\mathbf{C}_{n}$-inevitable elements by allowing these other elements. But only maximal elements are needed to obtain the lower bound. 


\section{Example: The TAll Fork}

The Tall Fork $F$ is a semigroup that was constructed by the second author in order to show that the Type I-Type II lower bound of 33 is not tight. A description of $F$ can be found at the beginning of [30, Section 4.14] and we shall follow the notation therein religiously. We also adjoin an identity $I$ to $F$ to make it a monoid $F^{I}$. Of course, $F$ and $F^{I}$ have the same complexity. The complexity of $F$ is at most 2 by the Depth Decomposition Theorem [41. We use our lower bound to show that the complexity of $F^{I}$ is at least 2. To do so, it will be convenient to use the following form of the "Tie-Your-Shoes" Lemma [30, Lemma 4.14.29].

Lemma 6.1 (Tie-Your-Shoes). Suppose $R$ is the distinguished $\mathscr{R}$-class of an $X$ generated group mapping monoid $M$. Assume that the $\mathscr{J}$-class $J$ of $R$ has Rees matrix coordinatization $J^{0} \cong \mathscr{M}^{0}(G, A, B, C)$. Let $a \in A$ correspond to the $\mathscr{R}$-class R. Suppose that $b_{1} C a_{0} \neq 0 \neq b_{2} C a_{0}$. Then if $(Y, P) \in \operatorname{SP}(M, X)$ is $\mathcal{F}_{n}$-closed and

$$
x=\left(a, g\left(b_{1} C a_{0}\right)^{-1}, b_{1}\right), y=\left(a, g\left(b_{2} C a_{0}\right)^{-1}, b_{2}\right) \in Y
$$

for some $g \in G$, then $x P y$.

Proof. Suppose that $P=\left\{B_{1}, \ldots, B_{r}\right\}$ and that $x \in B_{i}$ and $y \in B_{j}$ with $i \neq j$. Let $w \in X^{*}$ be a string mapping to the element $z=\left(a_{0}, 1, b_{1}\right)$. Note that $x z=$ $\left(a, g, b_{1}\right)=y z$. Proposition 5.15 then implies that $B_{i} z=B_{i} w$ and $B_{j} z=B_{j} w$ are disjoint, contradicting $\left(a, g, b_{1}\right) \in B_{i} z \cap B_{j} z$. Thus $i=j$, i.e., $x P y$.

We use all of $F$ as a generating set $X$ for $F^{I}$. Remember that elements of $\Omega(X)$ act via $\Upsilon$, which we omit from the notation. Set $L=\operatorname{SP}\left(F^{I}, X\right)$ and put $r=\left(a_{0}, 1,0^{\prime}\right)$. Let us begin by observing that $(r, r) \in \operatorname{States}_{0}(L)$. Let $\left(Y_{1}, P_{1}\right)=$ $(r, r) \cdot k^{\omega+*}$. It is easy to see that

$$
r \cdot k^{\omega+*}=r \cup K=\left\{\left(a_{0}, 1,0^{\prime}\right),\left(a_{0}, 1,2^{\prime}\right)\right\} .
$$

Hence Theorem 5.12 yields $Y_{1}=\left\{\left(a_{0}, 1,0^{\prime}\right),\left(a_{0}, 1,2^{\prime}\right)\right\}$. The Tie-Your-Shoes Lemma then implies that $P=\left\{Y_{1}\right\}$. Thus $\left(Y_{1},\left\{Y_{1}\right\}\right) \in \operatorname{States}_{0}(L)$. Let $\left(Y_{2}, P_{2}\right)=$ $\left(Y_{1},\left\{Y_{1}\right\}\right) \cdot t$. Proposition 5.15 then implies that

$$
Y_{2}=\left\{\left(a_{0},-1,0\right),\left(a_{0}, 1,2\right)\right\}
$$

and $P_{2}=\left\{Y_{2}\right\}$. Now consider $\left(Y_{3}, P_{3}\right)=\left(Y_{2},\left\{Y_{2}\right\}\right) \cdot h^{\omega+*}$. One easily verifies that $Y_{2} \cdot h^{\omega+*}=Y_{2} \cup H=\left\{a_{0}\right\} \times\{ \pm 1\} \times\{0,1,2,3\}$ and so Theorem 5.12 implies that $Y_{3}=\left\{a_{0}\right\} \times\{ \pm 1\} \times\{0,1,2,3\}$. Since

$$
\left(Y_{2},\left\{Y_{2}\right\}\right) \stackrel{\overline{h^{\omega+*}}}{\longrightarrow}\left(Y_{3}, P_{3}\right)
$$

is stable, we have

$$
\left(Y_{2},\left\{Y_{2}\right\}\right) \stackrel{\overline{h^{\omega}}}{\longrightarrow}\left(Y_{3}, P_{3}\right)
$$

and so $Y_{2}$ is contained in a single block of $P_{3}$. Repeated application of the TieYour-Shoes Lemma then establishes $P_{3}=\left\{Y_{3}\right\}$. Thus $\left(Y_{3},\left\{Y_{3}\right\}\right) \in \operatorname{States}_{0}(L)$ and hence

$$
\left(\left\{\left(a_{0}, 1,0\right),\left(a_{0},-1,0\right)\right\},\left\{\left\{\left(a_{0}, 1,0\right),\left(a_{0},-1,0\right)\right\}\right\}\right) \in \operatorname{States}_{0}(L) .
$$

Thus $F^{I}$ has complexity at least 2 by Theorem 5.18 . 


\section{REFERENCES}

[1] J. Almeida. Finite semigroups and universal algebra, volume 3 of Series in Algebra. World Scientific Publishing Co. Inc., River Edge, NJ, 1994. Translated from the 1992 Portuguese original and revised by the author. MR.1331143 (96b:20069)

[2] J. Almeida and B. Steinberg. On the decidability of iterated semidirect products with applications to complexity. Proc. London Math. Soc. (3), 80(1):50-74, 2000. MR 1719180 (2000j:20109)

[3] J. Almeida and B. Steinberg. Syntactic and global semigroup theory: a synthesis approach. In Algorithmic problems in groups and semigroups (Lincoln, NE, 1998), Trends Math., pages 1-23. Birkhäuser Boston, Boston, MA, 2000. MR1750489 (2001c:20120)

[4] C. J. Ash. Inevitable graphs: a proof of the type II conjecture and some related decision procedures. Internat. J. Algebra Comput., 1(1):127-146, 1991. MR.1112302 (92k:20114)

[5] K. Auinger. A new proof of the Rhodes type II conjecture. Internat. J. Algebra Comput., 14(5-6):551-568, 2004. International Conference on Semigroups and Groups in honor of the 65th birthday of Prof. John Rhodes. MR 2104770 (2005j:20068)

[6] B. Austin, K. Henckell, C. Nehaniv, and J. Rhodes. Subsemigroups and complexity via the presentation lemma. J. Pure Appl. Algebra, 101(3):245-289, 1995. MR.1348570 (97i:68140)

[7] T. A. Dowling. A class of geometric lattices based on finite groups. J. Combinatorial Theory Ser. B, 14:61-86, 1973. MR0307951 (46:7066)

[8] S. Eilenberg. Automata, languages, and machines. Vol. A. Academic Press, New York, 1974. Pure and Applied Mathematics, Vol. 58. MR0530382 (58:26604a)

[9] S. Eilenberg. Automata, languages, and machines. Vol. B. Academic Press, New York, 1976. With two chapters ("Depth decomposition theorem" and "Complexity of semigroups and morphisms") by Bret Tilson, Pure and Applied Mathematics, Vol. 59. MR0530382 (58:26604a)

[10] K. Henckell. Pointlike sets: the finest aperiodic cover of a finite semigroup. J. Pure Appl. Algebra, 55(1-2):85-126, 1988. MR968571 (90f:20094)

[11] K. Henckell, S. W. Margolis, J.-E. Pin, and J. Rhodes. Ash's type II theorem, profinite topology and $\mathrm{Mal}^{\prime} \mathrm{cev}$ products. I. Internat. J. Algebra Comput., 1(4):411-436, 1991. MR.1154442 (93h:20064)

[12] K. Henckell, J. Rhodes, and B. Steinberg. Aperiodic pointlikes and beyond. Internat. J. Algebra Comput., 20(2):287-305, 2010. MR2646752

[13] K. Henckell, J. Rhodes, and B. Steinberg. A profinite approach to stable pairs. Internat. J. Algebra Comput., 20(2):269-285, 2010. MR2646751 (2011d:20111)

[14] J. Karnofsky and J. Rhodes. Decidability of complexity one-half for finite semigroups. Semigroup Forum, 24(1):55-66, 1982. MR645703 (83d:20045)

[15] K. Krohn and J. Rhodes. Algebraic theory of machines. I. Prime decomposition theorem for finite semigroups and machines. Trans. Amer. Math. Soc., 116:450-464, 1965. MR 0188316 $(32: 5755)$

[16] K. Krohn and J. Rhodes. Complexity of finite semigroups. Ann. of Math. (2), 88:128-160, 1968. MR0236294(38:4591)

[17] K. Krohn, J. Rhodes, and B. Tilson. Algebraic theory of machines, languages, and semigroups. Edited by Michael A. Arbib. With a major contribution by Kenneth Krohn and John L. Rhodes. Academic Press, New York, 1968. Chapters 1, 5-9.

[18] S. W. Margolis. $k$-transformation semigroups and a conjecture of Tilson. J. Pure Appl. Algebra, 17(3):313-322, 1980. MR579091 (82a:20076)

[19] S. W. Margolis and B. Tilson. An upper bound for the complexity of transformation semigroups. J. Algebra, 73(2):518-537, 1981. MR640046 (84h:20068)

[20] J. McCammond, J. Rhodes, and B. Steinberg. Geometric semigroup theory. Internat. J. Algebra Comput., to appear.

[21] J. Rhodes. The fundamental lemma of complexity for arbitrary finite semigroups. Bull. Amer. Math. Soc., 74:1104-1109, 1968. MR0232873 (38:1196)

[22] J. Rhodes. Algebraic theory of finite semigroups. Structure numbers and structure theorems for finite semigroups. In K. Folley, editor, Semigroups (Proc. Sympos., Wayne State Univ., Detroit, Mich., 1968), pages 125-162. Academic Press, New York, 1969. MR0281817 (43:7531)

[23] J. Rhodes. Proof of the fundamental lemma of complexity (weak version) for arbitrary finite semigroups. J. Combinatorial Theory Ser. A, 10:22-73, 1971. MR0274615 (43:378) 
[24] J. Rhodes. Axioms for complexity for all finite semigroups. Advances in Math., 11(2):210-214, 1973.

[25] J. Rhodes. Proof of the fundamental lemma of complexity (strong version) for arbitrary finite semigroups. J. Combinatorial Theory Ser. A, 16:209-214, 1974. MR0338235 (49:3001)

[26] J. Rhodes. Kernel systems - a global study of homomorphisms on finite semigroups. J. Algebra, 49(1):1-45, 1977. MR0463337 (57:3290)

[27] J. Rhodes. Flows on automata. Technical report, Center for Pure \& Applied Mathematics, University of California, Berkeley, CA 94720-3840, 1995.

[28] J. Rhodes and B. Steinberg. Krohn-Rhodes complexity pseudovarieties are not finitely based. Theor. Inform. Appl., 39(1):279-296, 2005. MR2132592 (2006a:20106)

[29] J. Rhodes and B. Steinberg. Complexity pseudovarieties are not local; type II subsemigroups can fall arbitrarily in complexity. Internat. J. Algebra Comput., 16(4):739-748, 2006. MR2258836 (2007f:20100)

[30] J. Rhodes and B. Steinberg. The q-theory of finite semigroups. Springer Monographs in Mathematics. Springer, New York, 2009. MR2472427 (2010h:20132)

[31] J. Rhodes and B. Tilson. Local complexity of finite semigroups. In Algebra, topology, and category theory (collection of papers in honor of Samuel Eilenberg), pages 149-168. Academic Press, New York, 1976. MR0432792 (55:5775)

[32] J. Rhodes and B. R. Tilson. Lower bounds for complexity of finite semigroups. J. Pure Appl. Algebra, 1(1):79-95, 1971. MR0285649 (44:2867)

[33] J. Rhodes and B. R. Tilson. Improved lower bounds for the complexity of finite semigroups. J. Pure Appl. Algebra, 2:13-71, 1972. MR0299708 (45:8756)

[34] L. Ribes and P. A. Zalesskii. On the profinite topology on a free group. Bull. London Math. Soc., 25(1):37-43, 1993. MR 1190361 (93j:20062)

[35] B. Steinberg. On aperiodic relational morphisms. Semigroup Forum, 70(1):1-43, 2005. MR2107191 (2006c:20116)

[36] J. P. Stiffler. Extension of the fundamental theorem of finite semigroups. Advances in Math., 11(2):159-209, 1973.

[37] B. Tilson. Decomposition and complexity of finite semigroups. Semigroup Forum, 3(3):189250, 1971/72. MR0296201(45:5262)

[38] B. Tilson. Complexity of two $\mathscr{J}$-class semigroups. Advances in Math., 11(2):215-237, 1973.

[39] B. Tilson. On the complexity of finite semigroups. J. Pure Appl. Algebra, 5:187-208, 1974. MR0364517 (51:771)

[40] B. Tilson. Complexity of semigroups and morphisms, chapter XII, pages 313-384. In Eilenberg [9] 1976.

[41] B. Tilson. Depth decomposition theorem, chapter XI, pages 287-312. In Eilenberg 9], 1976.

[42] B. Tilson. Categories as algebra: an essential ingredient in the theory of monoids. J. Pure Appl. Algebra, 48(1-2):83-198, 1987. MR915990(90e:20061)

[43] B. Tilson. Type II redux. In Semigroups and their applications (Chico, Calif., 1986), pages 201-205. Reidel, Dordrecht, 1987. MR900660 (88k:20086)

[44] B. R. Tilson. Appendix to "Algebraic theory of finite semigroups". On the $p$-length of $p$ solvable semigroups: Preliminary results. In K. Folley, editor, Semigroups (Proc. Sympos., Wayne State Univ., Detroit, Mich., 1968), pages 163-208. Academic Press, New York, 1969. MR0277636 (43:3369)

Department of Mathematics/Computer Science, New College of Florida, 5800 Bay Shore Road, Sarasota, Florida 34243-2109

E-mail address: KHenckell@ncf.edu

Department of Mathematics, University of California at Berkeley, Berkeley, CalIFORNIA 94720

E-mail address: jrhodes@math.berkeley.edu

School of Mathematics and Statistics, Carleton University, 1125 Colonel By Drive, Ottawa, Ontario Canada K1S 5B6

E-mail address: bsteinbg@math.carleton.ca

Current address: Department of Mathematics, City College of New York, NAC 8/133, Convent

Avenue at 138th Street, New York, New York 10031

E-mail address: bsteinberg@ccny.cuny.edu 